\title{
Gestão de ciclo de vida e desenvolvimento de produto: análise bibliométrica e classificação da literatura
}

\author{
Angelo Varandas Junior ${ }^{2 *}$, Paulo Augusto Cauchick Miguel ${ }^{\mathrm{b}}$, \\ Marly Monteiro de Carvalhoc, Eduardo de Senzi Zancul ${ }^{\mathrm{d}}$ \\ a*avarandas@usp.br, Poli/USP, Brasil \\ bcauchick@usp.br, Poli/USP, Brasil \\ 'marlymc@usp.br, Poli/USP, Brasil \\ dezancul@usp.br, Poli/USP, Brasil
}

\begin{abstract}
Resumo
O objetivo deste trabalho é realizar um mapeamento e classificação da literatura, bem como uma análise bibliométrica sobre os conceitos Product Life Cycle Management (PLM), Product Development Process (PDP), Environmental Sustainability (ES) e suas interfaces. As publicações de interesse foram localizadas por meio de consultas na base de dados de periódicos da ISI Web of Knowledge, por meio do portal da CAPES, considerando publicações entre 2006 e 2010. Os resultados indicam que os trabalhos são publicados em uma gama variada de periódicos e congressos e que a maioria das publicações analisadas utiliza como abordagem metodológica o estudo de caso, mas também existe uma grande proporção de trabalhos teórico-conceituais. Quanto à natureza dos dados verificou-se que a abordagem qualitativa tem sido mais adotada e é praticamente predominante a condução de estudos descritivos. Em síntese, os trabalhos analisados incorporam o conceito de sustentabilidade ambiental e PLM no PDP e são mais aplicados em empresas, cadeia de suprimentos e desenvolvimento de software. De modo geral, os trabalhos analisados enfatizam a melhoria da gestão do PDP, o aumento do desempenho e a integração de informações de diferentes áreas e sistemas. A literatura converge para a inserção dos conceitos de sustentabilidade ambiental e PLM nas atuais práticas do PDP. Palavras-chave
\end{abstract}

Sustentabilidade ambiental. Gerenciamento do ciclo de vida de produtos. Processo de desenvolvimento de produtos.

\section{Introdução}

A competição no mercado global vem aumentando a preocupação das empresas quanto ao meio ambiente, o que leva essas empresas a inserirem aspectos de sustentabilidade ambiental no desenvolvimento de novos produtos. Nesse contexto, aspectos tais como a destinação dos produtos no fim de sua vida útil e o reaproveitamento deles, por meio de estratégias de fim de vida (tais como a remanufatura, reciclagem e reuso), vêm sendo focados pelas empresas. Além disso, devido às mudanças climáticas e consumo crescente de matérias-primas e recursos energéticos, a sustentabilidade é vista como um elemento chave para o desenvolvimento sustentável. Ao lado de aspectos econômicos, as dimensões ecológicas e sociais têm adquirido um maior significado (Bakshi \& Fiksel, 2003; Lindahl et al., 2003).

De modo geral, existem nas empresas possibilidades de redução na utilização dos recursos energéticos necessários para a produção. Medidas como reaproveitamento dos materiais utilizados na fabricação dos produtos proporcionam limitações no uso de matérias-primas e da energia na conversão da matériaprima reciclada (Van Berkel, 2007). A problemática da sustentabilidade tem como característica o seu caráter sistêmico, que coloca fortes desafios conceituais e operacionais, sendo necessário um esforço conjunto de todas as áreas da empresa. São tratadas nesse contexto muitas áreas de conhecimento que, a 
princípio, parecem distintas, mas cuja integração viabiliza a sustentabilidade (Krause et al., 2006; Cooper, 2007; Ebert \& De Man, 2008).

Entre as iniciativas existentes tem-se a abordagem do gerenciamento do ciclo de vida dos produtos ou Product Life Cycle Management (PLM), que envolve um grande número de processos de negócio que as empresas empregam para se manterem competitivas, tais como (Rozenfeld et al., 2006): i) Pesquisa \& Desenvolvimento; ii) Desenvolvimento de produto; iii) Gestão de configuração das informações do produto e processo; iv) Acompanhamento do produto depois do lançamento e sua retirada do mercado; e v) Gestão dos processos de negócios (BPM - Business Process Management). Nesse sentido, o PLM permite considerar a sustentabilidade na criação e desenvolvimento de um produto com o objetivo de diminuir o impacto ambiental e social de sua produção, uso e descarte. Além do mais, os processos de negócio de PLM, que ocorrem após o desenvolvimento e durante a sua produção, garantem que as especificações de desenvolvimento sejam atendidas e que os processos de negócio que lidam com os produtos possam ser melhorados constantemente de forma cíclica e gradual (Kilmann, 1995).

Alinhado aos amplos conceitos de PLM e sustentabilidade, destaca-se o Processo de Desenvolvimento de Produto (PDP), que é essencial na construção e sustentação de vantagem competitiva da empresa para fortalecer sua posição mercadológica. Esse processo consiste em um conjunto de atividades para definir novos produtos, em que alternativas são identificadas e avaliadas com critérios previamente definidos (Rozenfeld et al., 2006). Sua função é integrar todos esses critérios e otimizá-los, considerando as restrições existentes nos produtos, processos e organizacionais e, também, de geração de custos com retrabalhos (Clark \& Fujimoto, 1991). Também é condição desejável para a competitividade a inserção do enfoque mais específico da sustentabilidade ambiental.

Pelos motivos anteriormente citados, constata-se a importância da realização de estudos que busquem compreender os conceitos de PLM, PDP e sustentabilidade ambiental e suas interfaces. Assim sendo, o presente trabalho objetiva realizar uma classificação e codificação da literatura, por meio de uma análise bibliométrica de trabalhos relevantes sobre esses temas publicados nos últimos cinco anos. 0 propósito é também verificar quais são os trabalhos e autores mais importantes e os temas centrais pesquisados até o momento, visando identificar possíveis tendências e lacunas dos trabalhos sobre os três conceitos. Visando estabelecer os métodos e as técnicas de pesquisa empregadas para a consecução dos objetivos do presente trabalho, esses são apresentados na sequência.

\section{Métodos adotados na presente pesquisa}

Este trabalho é caracterizado como teóricoconceitual, mais especificamente voltado ao mapeamento e classificação da literatura sobre os conceitos de PLM, PDP e sustentabilidade ambiental. 0 presente trabalho não necessariamente se caracteriza como uma revisão tradicional da literatura (literature review), pois seu propósito não é a realização de uma análise crítica e nem comparativa entre as publicações selecionadas, mas visa identificar trabalhos relevantes, bem como lacunas de pesquisa existentes na literatura.

A revisão sistemática de literatura pode ser conduzida de diversas formas e mesclar técnicas qualitativas e quantitativas. Uma revisão sistemática tem como propósito identificar de forma abrangente e sintetizar os resultados de pesquisas sobre um tema em particular, por meio de procedimentos organizados, de modo transparente e replicável em cada etapa do processo de revisão (Littell et al., 2008). Neste trabalho utilizaram-se técnicas de bibliometria, análise de redes e classificação. As técnicas de bibliometria permitiram apresentar a evolução da literatura e das citações ao longo do tempo (lkpaahindi, 1985). As redes sociais foram utilizadas na análise das palavras chave, com auxílio do software Ucinet for Windows (ver Borgatti et al., 2002; Lopes \& Carvalho, 2012). A classificação permitiu tabular os trabalhos sobre diferentes aspectos como, por exemplo, o tipo de abordagem metodológica de pesquisa usando referenciais existentes (e.g. Martín et al., 1999; Carnevalli \& Cauchick Miguel, 2007).

\subsection{Protocolo de busca}

É importante destacar que, para identificar, localizar e recuperar as publicações de interesse foi consultada a base de dados ISI Web of Knowledge, por meio do portal da Coordenação de Aperfeiçoamento de Pessoal de Nível Superior (CAPES). Essa base foi selecionada pois nela é possível localizar todos os periódicos indexados cujo fator de impacto é calculado no Journal Citation Report (JCR), independentemente de sua base de origem (Lopes \& Carvalho, 2012).

$\mathrm{Na}$ consulta dos periódicos, buscaram-se como palavras-chave os seguintes termos (sem aspas e sem refinamento por área de conhecimento): PLM, PDP e environmental sustainability (sustentabilidade ambiental ou citado ao longo do texto pela sigla ES). Realizou-se a busca nos abstracts e, quando essa primeira opção não estava disponível na base 
consultada, realizou-se a busca no texto completo. 0 recorte temporal do estudo considerou trabalhos publicados nos últimos cinco anos (2006-2010), porém foram analisados os trabalhos publicados até março de 2010, período final da busca e análise. Inicialmente, foram encontrados 1.070 estudos para o termo PLM, 14.611 estudos para o termo PDP e 5.582 estudos para o termo ES. Em seguida foram realizadas buscas combinando os termos no banco de dados e foram encontrados 242 estudos para os termos PLM e PDP, 163 estudos para os termos PDP e ES, 61 estudos para os termos PLM e ES e apenas 13 estudos para os três termos juntos PLM, PDP e ES. Os resultados da busca combinada e refinamento são detalhados na Tabela 1.

Nessa primeira fase, foram identificados 479 estudos combinando os termos PLM, PDP e sustentabilidade ambiental, conforme ilustrado na Figura 1.

Ao retirar as publicações não disponíveis com acesso livre, aquelas referentes a patentes, as publicações duplicadas e as publicações que, pela leitura do abstract e/ou do texto completo, não estavam relacionadas aos temas de pesquisa (PLM, PDP e ES), resultaram 87 publicações para análise e classificação quanto ao propósito do trabalho de pesquisa. Para a análise dos dados, foram considerados os artigos em periódicos, trabalhos de congressos e simpósios. Não foram considerados livros, dissertações e teses, pelo fato de o portal da CAPES divulgar um número relativamente limitado desses tipos de publicação.

\subsection{Protocolo de classificação dos trabalhos}

Cada trabalho foi individualmente registrado por fichamento, utilizando-se uma adaptação de um formulário usado no levantamento de Carnevalli \& Cauchick Miguel (2007). Os trabalhos foram então classificados por: tipo de abordagem metodológica (modelagem, teórico-conceitual, revisão da literatura, simulação, survey, estudo de caso, pesquisa-ação e

Tabela 1. Estratificação do refinamento da busca combinada dos termos PLM, PDP e ES.

\begin{tabular}{|c|c|c|c|c|}
\hline Tópicos & (242) PLM e PDP & (163) PDP e ES & (61) PLM e ES & (13) PLM, PDP e ES \\
\hline Categoria & $\begin{array}{l}\text { (50) Gerenciamento, } \\
\text { (44) Pesquisa de operação, } \\
\text { (35) Engenharia industrial, } \\
\text { (33) Ciência ambiental, } \\
\text { (31) Engenharia ambiental, } \\
\text { (49) outros }\end{array}$ & $\begin{array}{l}\text { (51) Ciência ambiental, } \\
\text { (38) Engenharia ambiental, } \\
\text { (13) Estudos ambientais, } \\
\text { (10) Ecologia, } \\
\text { (10) Engenharia industrial, } \\
\text { (41) outros }\end{array}$ & $\begin{array}{l}\text { (27) Ciência ambiental, } \\
\text { (22) Engenharia ambiental, } \\
\text { (6) Tecnologia de construção, } \\
\text { (6) Gerenciamento }\end{array}$ & $\begin{array}{l}\text { (4) Engenharia ambiental, } \\
\text { (4) Ciência ambiental, } \\
\text { (3) Tecnologia de construção, } \\
\text { (2) Gerenciamento }\end{array}$ \\
\hline Área & $\begin{array}{l}\text { (122) Engenharia, } \\
\text { (68) Ciência da computação, } \\
\text { (56) Economia e negócios, } \\
\text { (44) Pesquisa de operação, } \\
\text { (36) Ciência ambiental e } \\
\text { ecológica }\end{array}$ & $\begin{array}{l}\text { (67) Engenharia, } \\
\text { (60) Ciência ambiental e } \\
\text { ecológica, } \\
\text { (12) Economia e negócios, } \\
\text { (12) Química, } \\
\text { (12) outros }\end{array}$ & $\begin{array}{l}\text { (30) Engenharia, } \\
\text { (28) Ciência ambiental e } \\
\text { ecológica, } \\
\text { (8) Economia e negócios, } \\
\text { (6) Tecnologia de construção }\end{array}$ & $\begin{array}{l}\text { (7) Engenharia, } \\
\text { (5) Ciência ambiental e } \\
\text { ecológica, } \\
\text { (3) Tecnologia de construção, } \\
\text { (2) Economia e negócios }\end{array}$ \\
\hline Documento & $\begin{array}{l}\text { (175) Artigo, } \\
\text { (69) Congresso, } \\
\text { (7) Revisão }\end{array}$ & $\begin{array}{l}\text { (135) Artigo, } \\
\text { (30) Congresso, } \\
\text { (10) Revisão }\end{array}$ & $\begin{array}{l}\text { (49) Artigo, } \\
\text { (10) Congresso, } \\
\text { (3) Revisão }\end{array}$ & $\begin{array}{l}\text { (10) Artigo, } \\
\text { (1) Material editorial, } \\
\text { (1) Congresso, } \\
\text { (1) Revisão }\end{array}$ \\
\hline Autor & $\begin{array}{l}\text { (26) IEEE, } \\
\text { (6) ASME, } \\
\text { (2) ACM, } \\
\text { (2) Dumslaff U., } \\
\text { (2) Ebert C., } \\
\text { (204) outros }\end{array}$ & $\begin{array}{l}\text { (4) IEEE, } \\
\text { (3) Luttropp C., } \\
\text { (3) ASME, } \\
\text { (2) Gantner U., } \\
\text { (2) Genaidy A., } \\
\text { (149) outros }\end{array}$ & $\begin{array}{l}\text { (2) Coltro L., } \\
\text { (2) Guelere A., } \\
\text { (2) IEEE, } \\
\text { (2) Kletecke R.M., } \\
\text { (2) Mourad A.L., } \\
\text { (51) outros }\end{array}$ & $\begin{array}{l}\text { (1) Schmidt, } \\
\text { (1) Cooper J.S., } \\
\text { (1) Van Schaik, } \\
\text { (10) outros }\end{array}$ \\
\hline $\begin{array}{l}\text { Ano de } \\
\text { publicação }\end{array}$ & $\begin{array}{l}\text { (55) } 2009, \\
(50) 2008, \\
(47) 2006, \\
(46) 2010, \\
(44) 2007\end{array}$ & $\begin{array}{l}\text { (47) } 2010, \\
\text { (31) } 2009, \\
\text { (31) } 2006, \\
\text { (31) } 2007, \\
\text { (23) } 2008\end{array}$ & $\begin{array}{l}\text { (21) } 2010, \\
\text { (16) } 2009, \\
\text { (12) } 2006, \\
\text { (9) } 2007 \\
\text { (3) } 2008\end{array}$ & $\begin{array}{l}\text { (6) } 2010 \\
\text { (3) } 2006, \\
\text { (2) } 2007 \\
\text { (2) } 2009\end{array}$ \\
\hline Idioma & $\begin{array}{l}\text { (238) lnglês, } \\
\text { (1) Espanhol, } \\
\text { (1) Croata, } \\
\text { (2) outros }\end{array}$ & $\begin{array}{l}\text { (154) lnglês, } \\
\text { (2) Alemão, } \\
\text { (2) Espanhol, } \\
\text { (5) outros }\end{array}$ & $\begin{array}{l}\text { (58) lnglês, } \\
\text { (2) Espanhol, } \\
\text { (1) Francês }\end{array}$ & $\begin{array}{l}\text { (12) lnglês, } \\
\text { (1) Espanhol }\end{array}$ \\
\hline $\begin{array}{l}\text { Fonte de } \\
\text { publicação }\end{array}$ & $\begin{array}{l}\text { (15) Int. Journal of Life Cycle } \\
\text { Assessment, } \\
\text { (4) European Journal of } \\
\text { Operational Research, } \\
\text { (4) Int. Journal of Production } \\
\text { Economics, } \\
\text { (219) outros }\end{array}$ & $\begin{array}{l}\text { (14) Journal of Cleaner } \\
\text { Production, } \\
\text { (8) International Journal of } \\
\text { Engineering Education, } \\
\text { (4) Int. Journal of Life Cycle } \\
\text { Assessment, } \\
\text { (137) outros }\end{array}$ & $\begin{array}{l}\text { (9) Int. journal of Life Cycle } \\
\text { Assessment, } \\
\text { (5) Journal of Cleaner } \\
\text { Production, } \\
\text { (3) Journal of Industrial } \\
\text { Ecology, } \\
\text { (44) outros }\end{array}$ & $\begin{array}{l}\text { (1) Int. Journal of } \\
\text { Engineering Education, } \\
\text { (3) Int. Journal of Life Cycle } \\
\text { Assessment, } \\
\text { (1) Journal of Management in } \\
\text { Engineering, } \\
\text { (8) outros }\end{array}$ \\
\hline
\end{tabular}


experimento), natureza da abordagem metodológica (qualitativa, quantitativa, descritiva e preditiva), abrangência do estudo (regional, nacional ou internacional), unidade de análise (pessoas, grupos, unidade organizacional e empresas), técnicas para a coleta de dados (questionário, entrevistas, análisedocumental, dados públicos e observação), e período analisado (retrospectivo, atual e longitudinal). Os trabalhos foram codificados conforme apresentado no Apêndice 1. Utilizaram-se o diagrama de afinidades e o diagrama em árvore para organizar e agrupar os dados de interesse dos artigos de forma hierárquica, classificando-os de acordo com o Apêndice 2: áreas de aplicação, benefícios alcançados e principais conceitos utilizados. Em função da grande dimensão dos diagramas, eles não são apresentados no presente trabalho.

\subsection{Protocolo da análise bibliométrica e análise de redes}

Como suporte para a análise bibliométrica utilizaram-se dois softwares: Sitkis (ver Schildt, 2002) e Ucinet (ver Borgatti et al., 2002). 0 Sitkis foi aplicado para importação e depuração (correção de erros) dos dados salvos em arquivo de texto do $1 S 1$ Web of Knowledge. 0 Ucinet auxiliou na

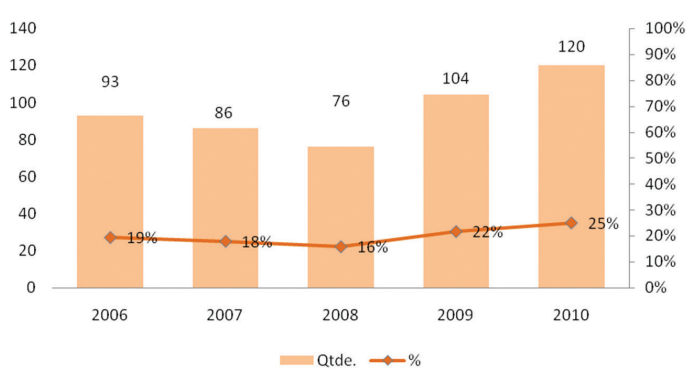

Figura 1. Publicações disponíveis no ISI Web of Knowledge para os termos escolhidos. elaboração das matrizes e mapas gráficos das redes de relacionamentos entre as palavras-chave e temas correlatos, conforme sugerido por Santos \& Bastos (2007), englobando um conjunto de técnicas para análise das redes sociais.

As redes sociais foram analisadas de duas formas: (i) do ponto de vista de suas características estruturais e (ii) do ponto de vista morfológico ou posicional dos atores. 0 ponto de vista estrutural examina a rede como um todo, enquanto o ponto de vista morfológico desce ao nível dos atores e suas ligações, focando nas relações entre os indivíduos e procurando compreender os papéis que os atores desempenham na manutenção ou expansão das redes. 0 levantamento de critérios estruturais abordou o tamanho, a densidade, as distâncias geodésicas e a coesão das redes.

A estrutura de uma rede de inter-relações de citações pode ser analisada com base em diversos indicadores, dependendo do objetivo pretendido com a análise. Assim, também foram utilizados indicadores de centralidade para interpretar algumas características das redes analisadas. Esses indicadores de centralidade permitem analisar a rede tanto no seu conjunto como individualmente, facilitando a compreensão da importância relativa de cada ator e relações dentro da rede. Com base na importância dos indicadores da rede, além dos indicadores de centralidade (grau de centralidade e índice de centralização), a presente análise utiliza ainda os seguintes indicadores nas redes de palavras-chave: densidade da rede, grau de intermediação e grau de proximidade. Na sequência, são apresentados os principais resultados do trabalho.

\section{Resultados: classificação dos trabalhos analisados}

Com a finalização da busca e organização bibliográfica, segundo os critérios previamente citados, foram analisados 87 trabalhos sobre os conceitos de PLM, PDP e sustentabilidade ambiental, publicados em

Tabela 2. Estratificação do refinamento da busca combinada dos termos PLM, PDP e ES.

\begin{tabular}{|c|c|c|c|c|c|c|}
\hline Periódicos & 2006 & 2007 & 2008 & 2009 & 2010 & Total \\
\hline Journal of Cleaner Production & 6 & 2 & 2 & 2 & & 12 \\
\hline International Journal of Life Cycle Assessment & 4 & & 2 & 2 & & 8 \\
\hline International Conference on PLM & & 3 & & & & 3 \\
\hline Conference on Life Cycle Engineering & & 2 & & & & 2 \\
\hline Concurrent Eng.-Research and Applications & & 1 & & 1 & & 2 \\
\hline European Journal of Operational Research & & 1 & & & 1 & 2 \\
\hline Inter. Journal of Computer Integ. Manuf. & & & 1 & 1 & & 2 \\
\hline Inter. Journal of Engineering Education & & 2 & & & & 2 \\
\hline International Journal of Production Economics & & & 2 & & & 2 \\
\hline Reliability Engineering \& System Safety & & 1 & & 1 & & 2 \\
\hline Total & 10 & 12 & 7 & 7 & 1 & 37 \\
\hline
\end{tabular}


25 diferentes periódicos e em congressos, referentes ao período de 2006 a 2010. Verificou-se que em apenas 15\% dos casos os periódicos apresentam mais de um artigo sobre o tema durante os cinco anos analisados, indicando que são dispersas as publicações sobre 0 assunto nos periódicos em geral, exceto em alguns como o Journal of Cleaner Production, que teve 12 artigos publicados, e o International Journal of Life Cycle Assessment, com oito artigos publicados. A Tabela 2 apresenta os periódicos e congressos que tiveram mais de um artigo publicado sobre o tema no período analisado e a Figura 2 mostra o percentual das publicações por ano analisadas neste trabalho.

Observa-se que existe uma oscilação pequena, mantendo-se em torno de 20 artigos publicados por ano no período analisado. A queda na quantidade de publicações analisadas para o ano de 2010 é devida, principalmente, à análise englobar as publicações até o mês de março de 2010. Comparando-se com dados da Figura 1, observa-se que, em geral, houve um aumento na quantidade de publicações sobre os três conceitos PDP, PLM e ES.

A Figura 3 classifica mais detalhadamente os trabalhos nos seguintes grupos de abordagens metodológicas de pesquisa: modelagem; teóricoconceitual; revisão da literatura; simulação; survey; estudo de caso; pesquisa-ação; e experimento, sendo que os valores indicados nas colunas referem-se à proporção de cada tipo de abordagem de pesquisa em relação ao total de trabalhos no período de cinco anos. Constata-se pela Figura 3 que o estudo de caso foi a abordagem metodológica mais adotada no período analisado e, em segundo lugar, destacam-se os trabalhos teórico-conceituais. Nota-se na Figura 3 que não foram encontrados trabalhos classificados como pesquisa-ação ou experimento, sendo esse último tipo um resultado esperado. Cabe destacar que algumas publicações foram classificadas em mais de um tipo de estudo por utilizarem abordagens de pesquisa multimétodo ou, ainda, na adoção de dada abordagem, serem realizados testes com aplicações reais ou um maior aprofundamento, tais como nas situações de modelagem, seguida por estudo de caso e survey complementada por um estudo de caso.

Sobre o apoio financeiro para as pesquisas, pouco mais de 26\% dos estudos indicaram a existência de algum tipo de apoio. No entanto, não é possível afirmar que a maioria das pesquisas são realizadas com recursos próprios, pelo fato de essa informação ser omitida nas publicações.

A Figura 4 apresenta a classificação dos trabalhos em relação à natureza das abordagens de pesquisa (quantitativa, qualitativa, descritiva e prescritiva), sendo que os valores das colunas indicam o percentual de

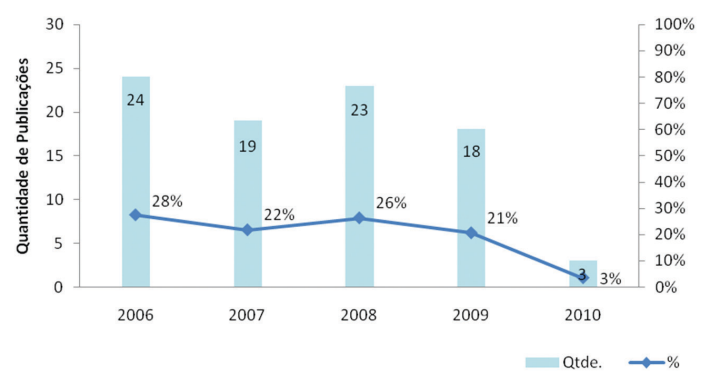

Figura 2. Distribuição percentual e quantidade de publicações por ano analisadas neste trabalho.

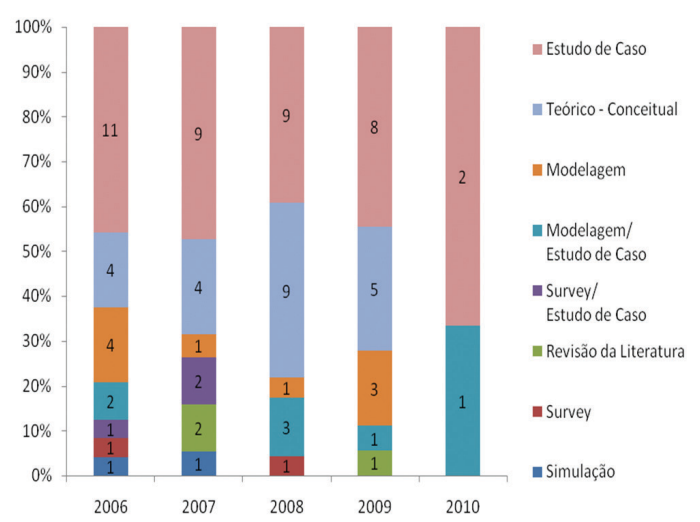

Figura 3. Classificação pelo tipo de abordagem de pesquisa nos estudos.

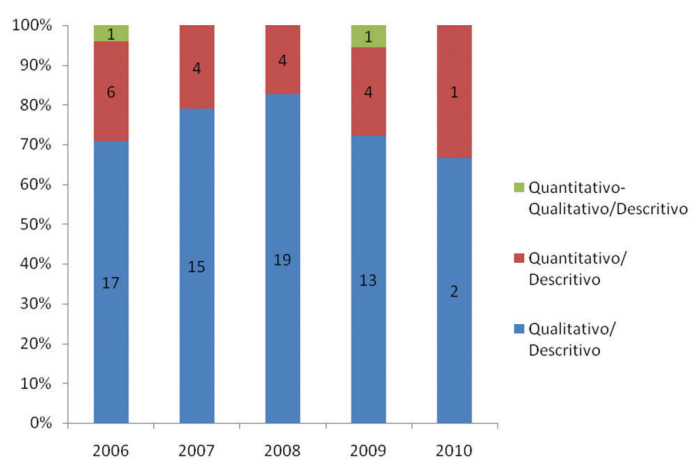

Figura 4. Classificação quanto à abordagem dos estudos.

ocorrência dessas abordagens em relação ao total de abordagens no período de cinco anos.

Verificou-se, portanto, que a abordagem de pesquisa qualitativa (76\%) tem sido mais adotada que a quantitativa $(24 \%)$ e que, basicamente, só estudos descritivos (100\%) foram realizados. Isso se deve, em parte, ao foco dos trabalhos ser, em geral, de caráter exploratório, pelo fato de os conceitos de PLM, PDP e sustentabilidade ambiental ainda não estarem plenamente consolidados na literatura (com base em estudos empíricos). Em relação aos 


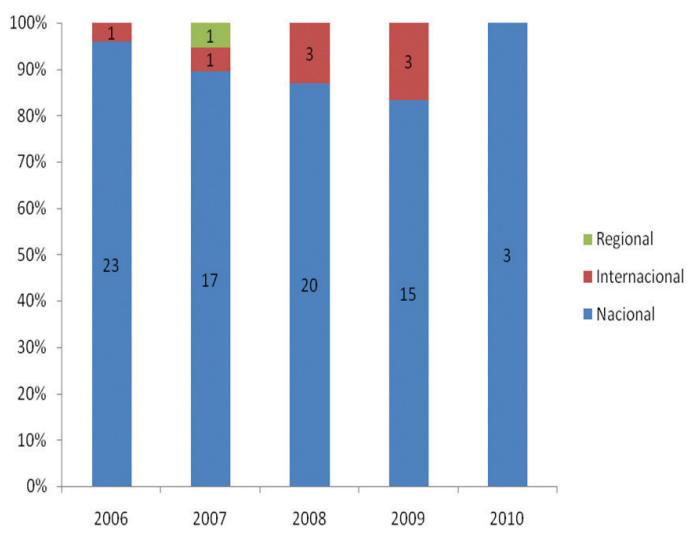

Figura 5. Classificação quanto à extensão dos estudos.

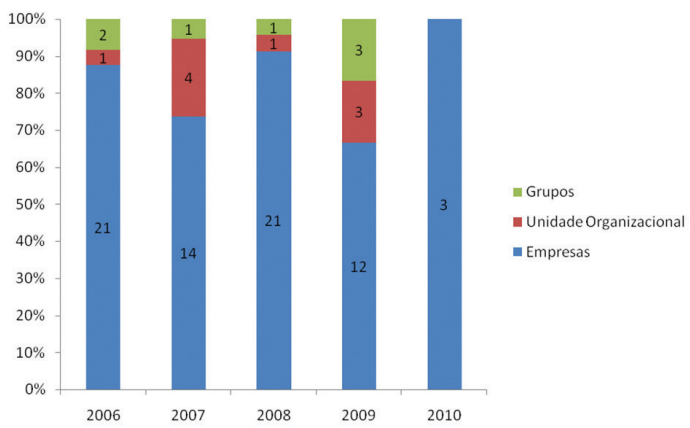

Figura 6. Classificação quanto à escolha do objeto de análise dos estudos. trabalhos que envolvem ambiente de análise regional, nacional ou internacional, a extensão é centralizada em ambiente nacional (90\%), conforme mostra a Figura 5. Sobre o objeto de análise dessas pesquisas, a maioria dos trabalhos considera empresas (81\%), como mostrado na Figura 6.

No caso da utilização de documentação direta e indireta para a coleta de dados (análise documental, dados públicos, revisão bibliográfica e observação), foram consideradas aquelas que foram indicadas nos trabalhos. Verificou-se que o uso conjunto de revisão bibliográfica e de análise documental ocorreu com maior frequência (57\%). Por questões óbvias, a revisão bibliográfica também corresponde a quase um quinto do total, seguida pela análise documental (7\%). Entretanto, outros tipos de documentação também foram adotados, como mostra a Figura 7, ressaltando-se que em dois terços das publicações (66\%) foi utilizado mais de um tipo de documentação.

Observou-se, que o estudo do tipo retrospectivo é o mais frequente, com mais da metade dos trabalhos analisados, como mostra a Figura 8, seguido do tipo atual (39\%) e, com menos frequência, do estudo longitudinal (8\%). Sobre os estudos de caso longitudinais (que analisam uma aplicação ao longo do tempo), verifica-se que existe uma carência desse tipo de estudo, que são relevantes para analisar a evolução dos conceitos PLM, PDP e sustentabilidade ambiental na extensão temporal.

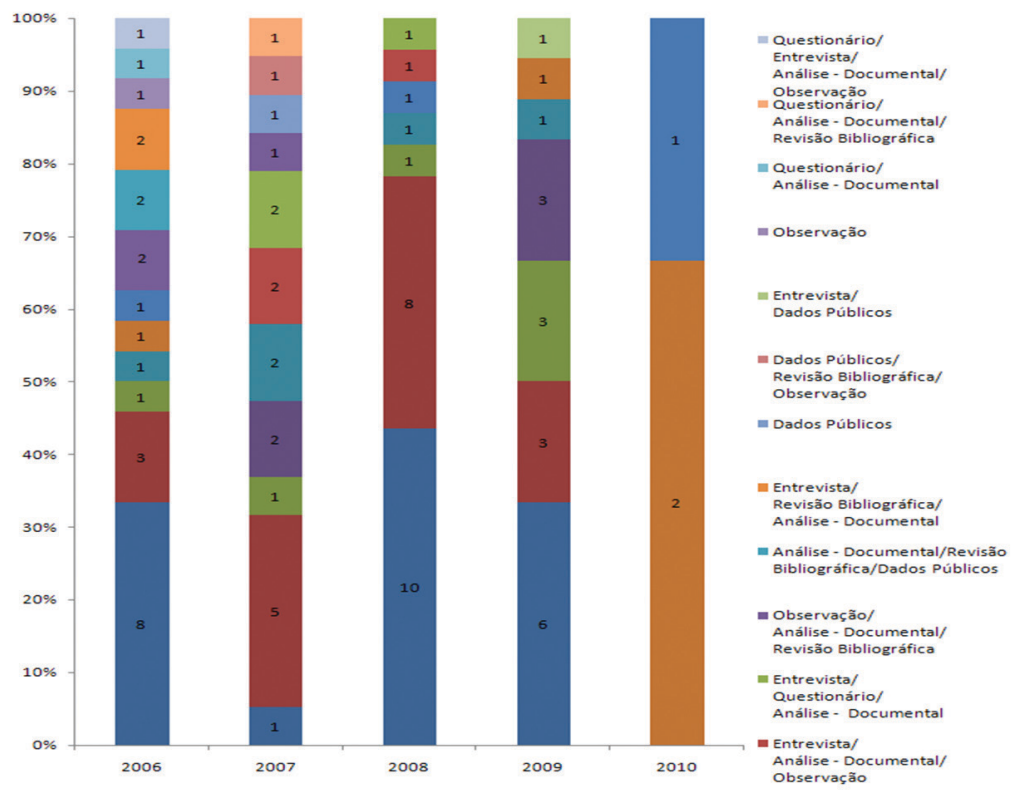

Figura 7. Distribuição da utilização de documentação para coleta de dados identificada nos estudos. 


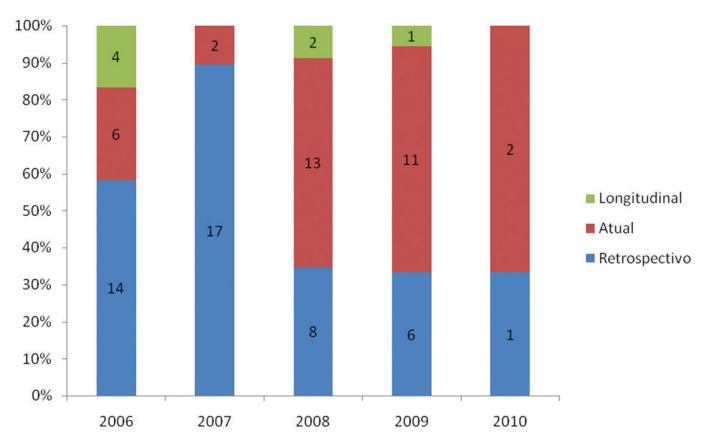

Figura 8. Classificação do período analisado nos estudos.

Com o uso do diagrama de afinidades e o diagrama em árvore foi possível organizar e agrupar os dados sobre o escopo das publicações por tipo (afinidades) e hierarquicamente (em árvore), visando analisar: (i) os conceitos de PLM, PDP e ES; (ii) os benefícios propostos pelas pesquisas; e (iii) as áreas de aplicação desses conceitos. Para elaboração desses diagramas foram seguidos os seguintes passos:

a) separação dos itens de interesse em planilhas do Excel ${ }^{\circledR}$, considerando os seguintes assuntos: escopo, definição e conceitos utilizados, benefícios, e áreas de aplicação;

b) desenvolvimento dos diagramas, separadamente, para cada assunto de interesse;

c) agrupamento das publicações por assuntos similares, mas mantendo as referências de todos os autores que os citaram;

d) determinação de um termo que representasse a ideia do grupo para cada um dos grupos, sendo que esse termo tinha um nível mais abstrato no primeiro nível;

e) montagem de tabela no Excel ${ }^{\circledR}$, obedecendo ao agrupamento realizado nas etapas anteriores e hierarquizando em níveis os termos do abstrato para o específico;

f) verificação dos níveis hierarquizados da tabela, se seus agrupamentos estavam consistentes - foram feitas as correções, quando necessário; e

g) revisão e finalização das tabelas com a análise de novos dados da literatura, respeitando os agrupamentos já realizados.

Sobre os conceitos utilizados nas publicações analisadas, verificou-se que $21 \%$ das publicações são sobre sustentabilidade ambiental (ES), que engloba o conceito Life Cycle Assessment (LCA); 21\% sobre PDP junto com sustentabilidade ambiental, que englobam os conceitos Design for Sustainability (DFS) e eco-design; 20\% sobre PLM, que engloba os conceitos End of Life (EOL), Life Cycle Costing (LCC) e Life Cycle Design (LCD); 16\% sobre PDP em conjunto com PLM, que englobam os conceitos Strategic Life Cycle Management (SLCM); 10\% sobre PDP; 10\% sobre PLM junto com sustentabilidade ambiental e $2 \%$ com os três conceitos juntos.

Nas conclusões das publicações são relatados alguns benefícios alcançados e/ou propostos com a inserção dos conceitos PLM e ES no PDP. Assim, identificou-se que 32\% referem-se a práticas de melhorias na gestão do PDP, 30\% investigaram variáveis que impactam no aumento de desempenho no PDP, 17\% buscam integrar informações de diferentes áreas e sistemas no PDP, 10\% envolvem suporte para a tomada de decisão pela alta administração no PDP, $8 \%$ buscam inserir conceitos e ferramentas de sustentabilidade no PDP, e 1\% para duas outras (alinhamento da cadeia de demanda e gestão do PLM; e melhoria do controle e planejamento de alternativas para desenvolvimento de novos produtos para software considerando o ciclo de vida dos produtos).

No caso das áreas de aplicação dos conceitos analisados neste trabalho foi verificado que 37\% estão relacionadas a ambientes complexos em termos de produto ou processo; $22 \%$, à gestão das diversas fases do PDP; 17\% são focadas, especificamente, na gestão de projetos e desenvolvimento de software; 11\%, na melhoria do desenvolvimento de novos produtos a partir das relações de colaboração na cadeia de suprimentos; 6\%, no desenvolvimento de tecnologia; $3 \%$, no estímulo a parcerias no codesenvolvimento; $2 \%$, na estruturação de programas de desenvolvimento educacional na área de engenharia; e 1\%, no controle e planejamento da cadeia de demanda.

\section{Resultados: conceitos PLM, PDP e ES e análise bibliométrica}

A estrutura de uma rede de inter-relações de citações pode ser analisada com base em diversos indicadores, dependendo do objetivo pretendido com a análise. Nesse sentido, também foram utilizados indicadores de centralidade para interpretar algumas características das redes. Esses indicadores de centralidade permitem analisar a rede tanto no seu conjunto como individualmente, facilitando a compreensão da importância relativa de cada ator e relações existentes na rede. Com base na importância dos indicadores da rede, além dos indicadores de centralidade (grau de centralidade e indice de centralização), a presente análise utiliza ainda os seguintes indicadores nas redes de palavraschave: densidade da rede, grau de intermediação e grau de proximidade. Na Tabela 3 é apresentada uma definição desses indicadores utilizados para análise. Os resultados decorrentes da combinação entre os 
conceitos (PDP e PLM; PDP e ES; PLM e ES; e PDP, PLM e ES) são apresentados na sequência.

\subsection{Análise bibliométrica}

Para realizar uma análise bibliométrica dos três conceitos juntos (PDP, PLM e ES) não foi utilizado critério de corte. Dessa forma, os trabalhos selecionados foram citados pelo menos uma vez e representam todas as citações sobre os termos PDP, PLM e ES, conforme mostra a Tabela 4.

Com base nos trabalhos listados na Tabela 4, verifica-se que a publicação mais citada foi a de Schmidt \& Butt (2006), que propõe ferramentas de gerenciamento da sustentabilidade no PDP considerando aspectos econômicos, sociais e ambientais. 0 trabalho de Van Schaik et al. (2010) trata de mudanças climáticas e gerenciamento de água e materiais e o trabalho de Cooper (2007) objetiva desenvolver conhecimentos interdisciplinares de sustentabilidade, Design for Environment (DFE) e Life Cycle Assessment (LCA).

Trabalhos sobre os conceitos PDP, PLM e ES combinados em pares, que foram os mais citados, apresentam tendência de crescimento. Porém, há poucos trabalhos sendo desenvolvidos que tratam dos três temas em conjunto. As Figuras 9 e 10 demonstram a quantidade absoluta e o percentual de citações por ano dos trabalhos mais citados sobre PLM e sustentabilidade ambiental.
Analisando as ligações entre as palavras-chave mostradas na Figura 11, observa-se que o tema sustainability está ligado diretamente a design for enviroment e recycling e, também, a sustainable development, que está relacionada ao tema design. 0 tema product development está ligado aos demais, exceto ao tema recycling.

Não foi possível encontrar uma rede de citações cruzadas e nem de cocitação para os trabalhos sobre os três temas PDP, PLM e ES selecionados. Para analisar as referências bibliográficas mais citadas, que compõem a base teórica dos trabalhos com respeito a PDP, PLM e ES selecionados, utilizaram-se como critério de corte as referências citadas mais de duas vezes. Com base nesse critério, obteve-se apenas uma publicação: Fleischmann et al. (2000).

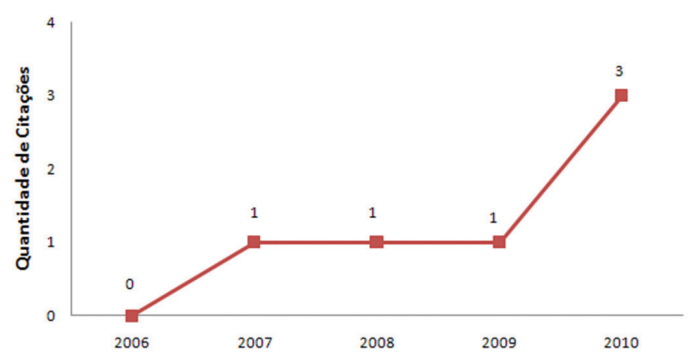

Figura 9. Número de citações por ano dos trabalhos sobre PDP, PLM e ES citados pelo menos uma vez. Nota: gráfico feito no MS Excel com os dados importados pelo software Sitkis.

Tabela 3. Definição dos termos utilizados na análise bibliométrica.

\begin{tabular}{|l|l|}
\hline \multicolumn{1}{|c|}{ Termo } & \multicolumn{1}{c|}{ Definição } \\
\hline Rede social & $\begin{array}{l}\text { A rede, constituída por conjunto de atores (nós da rede) que se relacionam (traços entre si) e visam comunicação, } \\
\text { troca e ajuda mútua, emerge a partir de interesses compartilhados e de situações vivenciadas. }\end{array}$ \\
\hline Ator da rede & Nós da rede, podem ser: artigos, autores, palavras-chave etc. \\
\hline Relacionamento & São as transações, interações e conexões entre esses diversos atores. \\
\hline Rede de citação cruzada & Permite identificar, dentro do conjunto de artigos, quais autores trabalham sobre temas correlatos. \\
\hline Rede de cocitação & $\begin{array}{l}\text { Mede a similaridade de citações entre os trabalhos, identificando o volume de trabalhos que citaram } \\
\text { conjuntamente os mesmos textos. }\end{array}$ \\
\hline Densidade da rede & $\begin{array}{l}\text { É o quociente de relações efetivamente existentes entre os atores das redes pelo total de ligações possíveis de } \\
\text { ocorrer. }\end{array}$ \\
\hline Grau de centralidade & $\begin{array}{l}\text { É a relação adjacente de um ator e pode ser dividida em grau de entrada (quantidade de ligações que um ator } \\
\text { recebe de outros atores) e grau de saída (quantidade de ligações que um ator estabelece com atores de um grupo). }\end{array}$ \\
\hline Grau de intermediação & $\begin{array}{l}\text { É a possibilidade que um ator tem de intermediar as comunicações entre pares de atores que não se relacionam } \\
\text { diretamente. }\end{array}$ \\
\hline Grau de proximidade & Avalia a proximidade ou distância de um ator em relação aos outros. \\
\hline Caminhos geodésicos & Caminho mais curto que um ator deve percorrer para se ligar a outros atores. \\
\hline
\end{tabular}

Tabela 4. Trabalhos citados pelo menos uma vez sobre PDP, PLM e ES.

\begin{tabular}{cccc}
\hline Autor & Revista & Citações \\
\hline Schmidt \& Butt (2006) & International Journal of Life Cycle Assessment & 4 \\
Van Schaik et al. (2010) & Minerals Engineering & 1 \\
Cooper (2007) & International Journal of Engineering Education & 1 \\
& Total & 6 \\
\hline
\end{tabular}




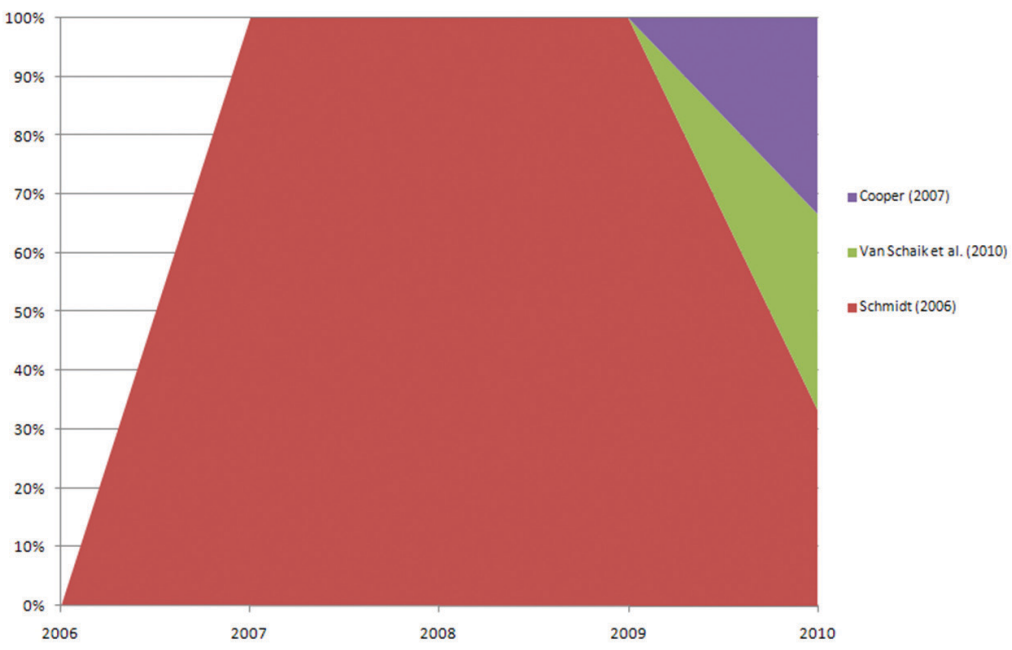

Figura 10. Percentual de citações por ano dos trabalhos sobre PDP, PLM e ES citados pelo menos uma vez. Nota: gráfico feito no MS Excel com os dados importados pelo software Sitkis.

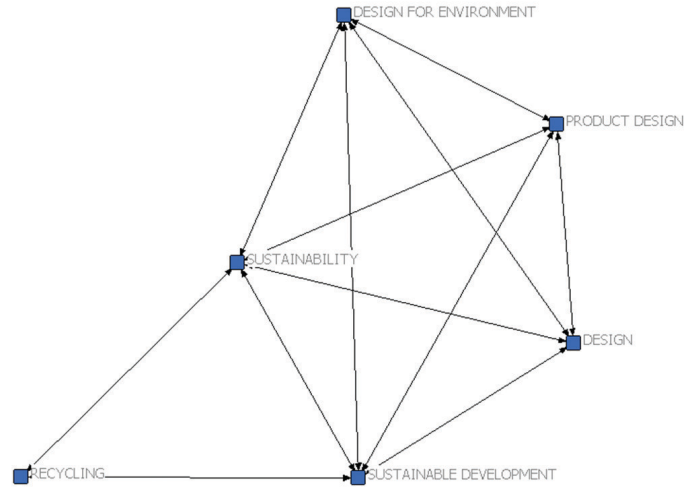

Figura 11. Rede de palavras-chave dos trabalhos sobre PDP, PLM e ES mais citados. Nota: gráfico feito no software Ucinet com os dados importados pelo software Sitkis.

Os autores relevantes em termos de quantidade de citações, para os trabalhos selecionados, nos temas de pesquisa foram: Fixson (2007) e Norris (2006), para os conceitos combinados de PDP e PLM; Pujari (2006) e Luttropp \& Lagerstedt (2006), para os conceitos de PDP e ES; Linton et al. (2007) e De Benedetto \& Klemes (2009), para os conceitos de PLM e ES; e Cooper (2007), Schmidt \& Butt (2006) e Van Schaik et al. (2010), para os três conceitos juntos.

$\mathrm{Na}$ análise da rede de interações das palavraschave dos conceitos PDP, PLM e ES obteve-se uma densidade da rede de $80 \%$, considerando-se que há um total de seis nós e 24 relações de 30 possíveis. Esse resultado demonstra uma alta conectividade da rede de palavras-chave para esses conceitos juntos.

0 grau de centralidade da rede é de 41,67\%, tanto para o grau de entrada como para o grau de saída. A média do grau de centralidade da rede (não simetrizada) foi de 5.714, indicando que, em média, cada palavra-chave se relaciona com outras seis. A Tabela 5 exibe o grau de entrada e grau de saída para todos os atores, indicando que a palavra-chave sustainability é central em termos de interações dessa rede, com um grau de entrada igual ao grau de saída de 10.000 (83,33\%). A Tabela 6 mostra alguns indicadores estatísticos da rede.

0 índice de centralização da rede é de 14,07\%, e indica um resultado de ausência de atores centrais. Para analisar a capacidade que os atores têm de intermediar a comunicação da rede, utilizaram-se o grau de intermediação e os indicadores estatísticos, mostrados na Tabela 7. As palavras-chave que obtiveram maior grau de intermediação foram: sustainability (17,77\%), sustainable development $(8,88 \%)$, design for environment $(8,88 \%)$ e recycling $(2,22 \%)$.

Para analisar a capacidade que os atores têm de relacionarem-se com os demais nós, avaliou-se o grau de proximidade. 0 grau de proximidade da rede é de $53,25 \%$ e a palavra sustainability teve o maior grau de proximidade, igual a 100.000 , e a menor distância geodésica, de 6.000. Esses resultados indicam que essa palavra possui uma melhor capacidade de relacionar-se com os demais atores. A Tabela 8 mostra a distância geodésica entre os atores, o grau de proximidade de todas as palavras-chave e os indicadores estatísticos.

\subsection{Principais tendências identificadas - sintese da análise de conteúdo}

Com base nos resultados e discussões anteriores, visando complementar a análise bibliométrica sobre PDP, ES e PLM e suas inter-relações, os seguintes 
Tabela 5. Grau de centralidade das palavras-chave sobre PDP, PLM e ES.

\begin{tabular}{|c|c|c|c|c|}
\hline Palavras-chave & Grau de saida & Grau de entrada & $\begin{array}{c}\text { Grau de saída } \\
\text { normalizado }(\%)\end{array}$ & $\begin{array}{l}\text { Grau de entrada } \\
\text { normalizado }(\%)\end{array}$ \\
\hline Sustainability & 10.000 & 10.000 & 83.333 & 83.333 \\
\hline Sustainable development & 7.000 & 7.000 & 58.333 & 58.333 \\
\hline Design for environment & 6.000 & 6.000 & 50.000 & 50.000 \\
\hline Design & 5.000 & 5.000 & 41.667 & 41.667 \\
\hline Recycling & 4.000 & 4.000 & 33.333 & 33.333 \\
\hline Product design & 4.000 & 4.000 & 33.333 & 33.333 \\
\hline
\end{tabular}

Tabela 6. Estatística descritiva do grau de intermediação das palavras-chave sobre PDP, PLM e ES.

\begin{tabular}{lcccc}
\multicolumn{1}{c}{ Estatistica descritiva } & Grau de saída & Grau de entrada & $\begin{array}{c}\text { Grau de saida } \\
\text { normalizado (\%) }\end{array}$ & $\begin{array}{c}\text { Grau de entrada } \\
\text { normalizado (\%) }\end{array}$ \\
\hline Mean & 5.714 & 5.714 & 47.619 & 47.619 \\
Std Dev & 2.050 & 2.050 & 17.087 & 17.087 \\
Sum & 40.000 & 40.000 & 333.333 & 333.333 \\
Variance & 4.204 & 4.204 & 291.950 & 291.950 \\
SSQ & 258.000 & 258.000 & 17.916 .666 & 17.916 .666 \\
MCSSQ & 29.429 & 29.429 & 2.043 .651 & 2.043 .651 \\
Euc Norm & 16.062 & 16.062 & 133.853 & 133.853 \\
Minimum & 4.000 & 4.000 & 33.333 & 33.333 \\
Maximum & 10.000 & 10.000 & 83.333 & 83.333 \\
Nof Obs & 7.000 & 7.000 & 7.000 & 7.000 \\
\hline
\end{tabular}

Tabela 7. Grau de intermediação das palavras-chave sobre PDP, PLM e ES.

\begin{tabular}{lcc}
\hline \multicolumn{1}{c}{ Palavras-chave } & $\begin{array}{c}\text { Grau de } \\
\text { intermediação }\end{array}$ & $\begin{array}{c}\text { Grau de } \\
\text { intermediação } \\
\text { normalizado (\%) }\end{array}$ \\
\hline Sustainability & 2.667 & 17.778 \\
Sustainable development & 1.333 & 8.889 \\
Design for environment & 1.333 & 8.889 \\
Recycling & 0.333 & 2.222 \\
Mean & 0.857 & 5.714 \\
Std Dev & 0.906 & 6.040 \\
Sum & 6.000 & 40.000 \\
Variance & 0.821 & 36.483 \\
SSQ & 10.889 & 483.951 \\
MCSSQ & 5.746 & 255.379 \\
Euc Norm & 3.300 & 21.999 \\
Minimum & 0.000 & 0.000 \\
Maximum & 2.667 & 17.778 \\
N of Obs & 7.000 & 7.000 \\
\hline
\end{tabular}

pontos-chave da análise de conteúdo foram sumarizados a partir das 87 publicações selecionadas na literatura.

0 tema PDP apresenta-se, em geral, diretamente ligado à necessidade de maior competitividade industrial, sendo esse um resultado esperado das ações de melhoria do PDP. Grande ênfase é dada à gestão do processo de desenvolvimento como um todo, envolvendo aspectos como sequência de atividades, seleção e adoção de métodos de projeto, otimização do fluxo de informações e melhoria organizacional, notadamente com maior esforço para viabilizar o trabalho em equipes multidisciplinares. 0 foco é em processos empregados para o desenvolvimento de produtos que considerem os requisitos de desempenho, de custos e também de impacto no meio ambiente. Nesse sentido são adotados métodos específicos de projeto, que têm como objetivo considerar, desde as fases iniciais do desenvolvimento do produto, as questões críticas que terão impactos somente mais tarde, nas fases posteriores do ciclo de vida.

A ES destaca a importância da inclusão da variável ambiental no processo de desenvolvimento, buscando minimizar o uso dos recursos naturais e reduzir os impactos ambientais inerentes à produção. Nos artigos que destacam esse tema, maior ênfase é dada à estimativa e à avaliação dos impactos ambientais que podem ser atribuídos a um produto ao longo de seu ciclo de vida - consumo de energia, emissões, entre outros aspectos. Nesse sentido, é empregada a LCA para estimar o impacto ambiental ao longo do ciclo de vida. Na aplicação da LCA são considerados principalmente os impactos ambientais gerados nas fases de produção, de uso e de descarte dos produtos. A fase de desenvolvimento é geralmente menos relevante, pois nela, tipicamente, os impactos no meio ambiente são comparativamente menores. No entanto, deve-se observar que as decisões de projeto realizadas na fase de desenvolvimento determinam grande parte dos impactos ambientais que ocorrem nas fases seguintes do ciclo de vida. Isso evidencia 
Tabela 8. Grau de proximidade das palavras-chave sobre PDP, PLM e ES.

\begin{tabular}{lcc}
\hline \multicolumn{1}{c}{ Palavras-chave } & Distância & $\begin{array}{c}\text { Grau de } \\
\text { proximidade }\end{array}$ \\
\hline Sustainability & 6.000 & 100.000 \\
Sustainable development & 7.000 & 85.714 \\
Design for environment & 7.000 & 85.714 \\
Design & 8.000 & 75.000 \\
Product design & 8.000 & 75.000 \\
Recycling & 9.000 & 66.667 \\
Mean & 7.714 & 79.252 \\
Std Dev & 1.030 & 11.129 \\
Sum & 54.000 & 554.762 \\
Variance & 1.061 & 123.849 \\
SSQ & 424.000 & 44.832 .766 \\
MCSSQ & 7.429 & 866.942 \\
Euc Norm & 20.591 & 211.737 \\
Minimum & 6.000 & 66.667 \\
Maximum & 9.000 & 100.000 \\
N of Obs & 7.000 & 7.000 \\
\hline
\end{tabular}

a inter-relação entre PDP e ES. No PDP, abordagens e métodos como o Design for Sustainability e o eco-design são empregados desde a conceituação dos produtos para direcionar as escolhas sobre a estrutura, os materiais e os processos de produção. 0 objetivo é antecipar o atendimento dos requisitos ambientais desde as etapas iniciais do desenvolvimento. Já a LCA é uma ferramenta que indica as consequências dessas escolhas de projeto no consumo de recursos e no meio ambiente.

O PLM destaca a necessidade de promover uma visão completa do ciclo de vida do produto, compreendendo todas as fases pelas quais o produto passa, desde a sua concepção até a destinação final, após o seu uso. 0 objetivo é possibilitar que requisitos das fases posteriores do ciclo de vida como, por exemplo, a facilidade de desmontagem para a reciclagem, sejam considerados desde as fases iniciais de conceituação. Viabilizar essa visão na prática requer a integração de informações de várias áreas e sistemas. A ênfase principal é no apoio à criação, à gestão, à disseminação e ao uso das informações de produtos ao longo do ciclo de vida. Dentre as informações de produtos geradas no ciclo de vida estão: listas de requisitos, estruturas de produto, modelos em CAD, planos de processo de fabricação, resultados de simulação, dentre outras. Idealmente, tais informações precisam estar atualizadas para serem acessadas ao longo de todo o ciclo de vida por todas as pessoas envolvidas e autorizadas. A integração de informações entre áreas e sistemas promovida pelo PLM contribui para a otimização do PDP e para que se possa atuar na redução dos impactos ambientais, conforme a ênfase dada pela ES.
Em síntese, os trabalhos que abordam o conceito de PLM consideram a visão ampla do ciclo de vida, desde a concepção até as etapas finais de reuso, remanufatura e reciclagem como estratégia para o fim da vida útil de produtos. Para os trabalhos que utilizam o conceito de sustentabilidade, o foco está na avaliação dos impactos no meio ambiente e o PDP busca desenvolver produtos com menor impacto ambiental, envolvendo princípios de eco-design, mas que tragam também benefícios econômicos e sociais, termo mais conhecido como Triple Bottom Line.

\section{Conclusões}

Primeiramente, cabe considerar que os objetivos inicialmente estabelecidos foram atingidos. Pelos resultados da análise e classificação da literatura constata-se que os trabalhos são publicados de forma dispersa em periódicos e congressos e que os periódicos que mais abordam o tema de pesquisa são o Journal of Cleaner Production e o International Journal of Life Cycle Assessment. Verifica-se, ainda, que a maioria das publicações analisadas utiliza como abordagem metodológica o estudo de caso, seguido por trabalhos teórico-conceituais. Em algumas publicações constatou-se a adoção de multimétodo. Quanto à natureza dos dados constata-se que a abordagem qualitativa tem sido mais adotada em relação à quantitativa e que somente estudos descritivos foram realizados, devido, em parte, ao fato de as publicações explorarem esses conceitos, em sua pauta de pesquisa, já há algum tempo, embora eles não estejam totalmente consolidados na literatura (notadamente PLM e ES). Constata-se ainda um grupo de autores considerados relevantes, pela quantidade de citações, com publicações de 2006 a 2010.

Sobre o objeto de análise dessas pesquisas, a maioria analisou empresas e empregou mais de um tipo de documentação para coleta de dados. Ainda, observa-se que o estudo do tipo retrospectivo é mais frequente que o longitudinal, sendo esse último muito importante para analisar a evolução dos conceitos PLM, PDP e ES. Os tópicos mais utilizados no desenvolvimento dos trabalhos analisados referem-se aos temas End of Life, Life Cycle Assessment, Life Cycle Costing, Life Cycle Design, Design for Sustainability, Eco-Design, Strategic Life Cycle Management, e Sustentabilidade.

Considerando a importância dos conceitos PDP, PLM e ES para as empresas obterem sucesso no mercado, conclui-se que, relativamente, ainda existe uma certa escassez de trabalhos publicados tratando dos três temas em conjunto, apesar de eles terem sido desenvolvidos já há algum tempo. Em síntese, os trabalhos analisados incorporam os conceitos ES 
e PLM no PDP e são mais aplicados em empresas, cadeias de suprimentos e desenvolvimento de software

Sobre os benefícios propostos por esta amostra de trabalhos analisados (87), de modo geral, enfatiza-se a melhoria da gestão do PDP, o aumento do desempenho e a integração de informações de diferentes áreas e sistemas. Além disso, é importante destacar que os trabalhos de relevância sobre os três conceitos abordam a inserção de ferramentas de gerenciamento da sustentabilidade ambiental no PDP, considerando aspectos econômicos, sociais e ambientais.

Os resultados do presente trabalho oferecem uma classificação da produção científica dos conceitos de gestão de ciclo de vida, desenvolvimento de produto e sustentabilidade ambiental e uma sumarização do conteúdo principal dessas publicações. Uma limitação do trabalho é a não realização de uma revisão crítica aprofundada da literatura sobre os conceitos analisados que, embora não tenha sido o propósito e escopo, seria bem-vinda em trabalhos futuros.

Nesse sentido, uma das propostas para trabalhos futuros é uma análise sobre a integração dos conceitos PDP, PLM e ES, delineando o contorno entre as interfaces, basicamente em função da escassez de estudos desse tipo. Além disso, devem-se aprofundar os estudos de análise bibliométrica para disseminar essa técnica na área de engenharia de produção.

Por fim, o trabalho conclui que a literatura converge para a inserção dos conceitos ES e PLM nas atuais práticas do PDP. Essa conclusão pode ser justificada com base na maior demanda de exigências ambientais pela sociedade e na necessidade de as organizações empregarem a estratégia de fim de vida dos produtos (remanufatura, reciclagem e reuso) para cumprir aspectos sociais, ambientais e econômicos exigidos pelo mercado. Esses argumentos são reforçados nas publicações analisadas. Entretanto, uma análise mais aprofundada é necessária para validar esse ponto conclusivo, considerando uma vertente empírica.

\section{Referências}

Aurich, J. C., Schweitzer, E., \& Fuchs, C. (2007). Life cycle management of industrial product-service systems. In Proceedings of the 14th CIRP Conference on Life Cycle Engineering, Tokyo, Japan.

Baccile, N., Babonneau, F., Thomas, B., \& Coradin, T. (2009). Introducing ecodesign in silica sol-gel materials. Journal of Materials Chemistry, 19(45), 8537-8559. http://dx.doi. $\operatorname{org} / 10.1039 / \mathrm{b} 911123 \mathrm{a}$

Bakshi, B. R., \& Fiksel, J. (2003). The Quest for Sustainability: challenges for process systems engineering. AlChe Journal, 49(6), 1350-1358. http://dx.doi.org/10.1002/ aic. 690490602
Barry, E. J., Kemerer, C. E., \& Slaughter, A. S. (2006). Environmental volatility, development decisions, and software volatility: a longitudinal analysis. Management Science, 52(3), 448-464. http://dx.doi.org/10.1287/ mnsc. 1050.0463

Belkadi, F., Troussier, N., Huet, F., Gidel, T., Bonjour, E., \& Eynard, B. (2008). Innovative PLM-based approach for collaborative design between OEM and suppliers: Case study of aeronautic industry. In Proceedings of the 2 nd IFIP Topical Session on Computer-Aided Innovation Held at the 20th World Computer Congress, Milan.

Ben Mahmoud-Jouini, S., \& Lenfle, S. (2010). Platform re-use lessons from the automotive industry. International Journal of Operations \& Production Management, 30(1), 98124. http://dx.doi.org/10.1108/01443571011012398

Bergea, 0., Karlsson, R., Hedlund-Astrom, A., Jacobsson, P., \& Luttropp, C. (2006). Education for sustainability as a transformative learning process: a pedagogical experiment in ecodesign doctoral education. Journal of Cleaner Production, 14(15-16), 1431-1442. http:// dx.doi.org/10.1016/j.jclepro.2005.11.020

Bertolini, M., Colacino, P., Delnevo, N., \& Petroni, A. (2007) Stakeholders' influence and internal championing of product stewardship in the ltalian food packaging industry. In Proceedings of the 4th International Conference on Product Lifecycle Management, Stezzano, Italy.

Bidokhti, N. (2008). The impact of reliability requirements on development life cycle. In Proceedings of the 54th Annual Reliability and Maintainability Symposium, Las Vegas.

Bordoloi, S., \& Guerrero, H. H. (2008). Design for control: A new perspective on process and product innovation. International Journal of Production Economics, 113(1), 346-358. http://dx.doi.org/10.1016/j. ijpe.2007.02.043

Broy, M. (2009). From system requirements documents to integrated system modeling artifacts. In Proceedings of the 9th ACM Symposium on Document Engineering, Munich, Germany.

Borgatti, S., Everett, M., \& Freeman, L. (2002). Ucinet for Windows: software for social network analysis. Analytic Technologies. PMCid:PMC99611.

Buganza, T., \& Verganti, R. (2006). Life-cycle flexibility: How to measure and improve the innovative capability in turbulent environments. Journal of Product Innovation Management, 23(5), 393-407. http://dx.doi.org/10.1111/ j.1540-5885.2006.00212.x

Cebon, P., Hauptman, 0., \& Shekhar, C. (2008) Product modularity and the product life cycle: new dynamics in the interactions of product and process technologies. International Journal of Technology Management, 42(4), 365-386. http://dx.doi.org/10.1504/ IJTM.2008.019381

Carnevalli, J. A., \& Cauchick Miguel, P. A. (2007). Revisão, análise e classificação da literatura sobre o QFD: tipos de pesquisa, dificuldades de uso e benefícios do método. Gestão \& Produção, 14(3), 557-579. http://dx.doi. org/10.1590/S0104-530X2007000300011

Chang, Y. C., Cheng, F. T., \& Wang, T. L. (2007). Novel semiconductor business model - Engineering chain for the semiconductor industry. In Proceedings of the IEEE International Conference on Robotics and Automation, Rome, ltaly. 
Ciolkowski, M., \& Soto, M. (2008). Towards a comprehensive approach for assessing open source projects. In Proceedings of the Joint Meeting of the International Workshop on Software Measurement (IWSM), Munich, Germany.

Clark, K. B., \& Fujimoto, T. (1991). Product development performance: strategy, organization and management in the world auto industry. Boston: Harvard Business School Press.

Cooper, J. S. (2007). Evolution of an interdisciplinary course in sustainability and design for environment. International Journal of Engineering Education, 23(2), 294-300.

Cooper, J., Godwin, C., \& Hall, E. S. (2008). Modeling process and material alternatives in life cycle assessments. International Journal of Life Cycle Assessment, 13(2), 115-123. http://dx.doi.org/10.1065/ lca2007.06.341

Cordella, M., Tugnoli, A., Spadoni, G., Santarelli, F., \& Zangrando, T. (2008). LCA of an Italian lager beer. International Journal of Life Cycle Assessment, 13(2), 133139. http://dx.doi.org/10.1065/lca2007.02.306

Da Silva, C. M., Loubach, D. S., \& Cunha, A. M. (2008). Applying the use case points effort estimation technique to avionics systems. In Proceedings of the IEEE/ AIAA 27th Digital Avionics Systems Conference, St Paul.

De Benedetto, L., \& Klemes, J. (2009). The environmental performance strategy map: an integrated LCA approach, to support the strategic decision-making process. Journal of Cleaner Production, 17(10), 900-906. http://dx.doi. org/10.1016/j.jclepro.2009.02.012

Dehghanian, F., \& Mansour, S. (2008). A framework for moving toward for sustainable supply chain management. In Proceedings of the 38th International Conference on Computers and Industrial Engineering, Beijing.

Donnelly, K., Beckett-Furnell, Z., Traeger, S., Okrasinski, T., \& Holman, S. (2006). Eco-design implemented through a product-based environmental management system. Journal of Cleaner Production, 14(15-16), 1357-1367. http://dx.doi.org/10.1016/j.jclepro.2005.11.029

Ebert, C., \& De Man, J. (2008). Effectively utilizing project, product and process knowledge. Information and Software Technology, 50(6), 579-594. http://dx.doi. org/10.1016/j.infsof.2007.06.007

Elghali, L., Clift, R., Begg, K. G., \& McLaren, S. (2008). Decision support methodology for complex contexts. Proceedings of the Institution of Civil EngineersEngineering Sustainability, 161(1), 7-22. http://dx.doi. org/10.1680/ensu.2008.161.1.7

Eun, J. H., Son, J. H., Moon, J. M., \& Chung, J. S. (2009). Integration of life cycle assessment in the environmental information system. International Journal of Life Cycle Assessment, 14(4), 364-373. http://dx.doi.org/10.1007/ s11367-009-0076-6

Fargnoli, M., \& Kimura, F. (2006). Screening life cycle modeling for sustainable product design. In Proceedings of the 12th CIRP International Conference on Life Cycle Engineering, Grenoble, France.

Fixson, S. K. (2007). Modularity and commonality research: past developments and future opportunities. Concurrent Engineering-Research and Applications, 15(2), 85-111. http://dx.doi.org/10.1177/1063293X07078935

Fleischmann, M., Krikke, H. R., \& Dekker, R. (2000). A characterisation of logistics networks for product recovery. Omega-International Journal of Management Science, 28(6), 653-666. http://dx.doi.org/10.1016/ S0305-0483(00)00022-0

Genaidy, A., \& Karwowski, W. (2008). A roadmap for a methodology to assess, improve and sustain intra- and inter-enterprise system performance with respect to technology-product life cycle in small and medium manufacturers. Human Factors and Ergonomics in Manufacturing, 18(1), 70-84. http://dx.doi.org/10.1002/ hfm.20097

Giudice, F., Ballisteri, F., \& Risitano, G. (2009). A concurrent design method based on DFMA-FEA integrated approach. Concurrent Engineering-Research and Applications, 17(3), 183-202. http://dx.doi. $\operatorname{org} / 10.1177 / 1063293 X 09343337$

Han, K. H., \& Do, N. (2006). An object-oriented conceptual model of a collaborative product development management (CPDM) system. International Journal of Advanced Manufacturing Technology, 28(7-8), 827-838. http://dx.doi.org/10.1007/s00170-004-2424-9

Hauschild, M. Z., Dreyer, L. C., \& Jorgensen, A. (2008). Assessing social impacts in a life cycle perspective - Lessons learned. CIRP Annals-Manufacturing Technology, 57(1), 21-24. http://dx.doi.org/10.1016/j.cirp.2008.03.002

Hobby, C., Rydell, N., Sjogren, E., \& Williams, W. (2009). IT products. Going beyond green - can high performance and sustainability co-exist? In Proceedings of the IEEE International Symposium on Sustainable Systems and Technology, Tempe.

Hochschorner, E., \& Finnveden, G. (2006). Life cycle approach in the procurement process: The case of defence materiel. International Journal of Life Cycle Assessment, 11(3), 200-208. http://dx.doi.org/10.1065/ lca2005.10.230

Huang, A. H. (2009). A model for environmentally sustainable information systems development. Journal of Computer Information Systems, 49(4), 114-121.

lkpaahindi, L. (1985). An Overview of Bibliometrics: its Measurements, Laws and their Applications. Libri, 35(2), 163-176.

Jawahir, 1. S., Rouch, K. E., Dillon, O. W., Holloway, L., \& Hall, A. (2007). Design for sustainability (DFS): New challenges in developing and implementing a curriculum for next generation design and manufacturing engineers. International Journal of Engineering Education, 23(6), 1053-1064.

Jiang, Z. G., Zhang, H., Fu, C., \& Jiang, D. R. (2008). The operation mode of green manufacturing based information technology. In Proceedings of the International Conference on Informational Technology and Environmental System Science, Jiaozuo, China.

Joore, P. (2008). The V-Cycle for system innovation translating a broad societal need into concrete product service solutions: the multifunctional centre Apeldoorn case. Journal of Cleaner Production, 16(11), 1153-1162. http://dx.doi.org/10.1016/j.jclepro.2007.08.007

Juttner, U., Godsell, J., \& Christopher, M. G. (2006). Demand chain alignment competence - delivering value through product life cycle management. In Proceedings of the 22nd IMP Conference, Milan, Italy.

Kennedy, G. A. L., Siemieniuch, C. E., Sinclair, M. A., Kirwan, B. A., \& Gibson, W. H. (2007). Proposal for a sustainable framework process for the generation, validation, and application of human reliability assessment within the 
engineering design lifecycle. Reliability Engineering \& System Safety, 92(6), 755-770. http://dx.doi. $\operatorname{org} / 10.1016 / j$.ress.2006.03.007

Khota, I., \& Pretorius, L. (2008). Intellectual property scorecard: Strategically capitalising on value created by innovation and R\&D. In Proceedings of the Portland International Conference on Management Engineering and Technology, Cape Town, South Africa.

Kilmann, R. (1995). A holistic program and critical success factors of corporate transformation. European Management Journal, 13(2), 175-186. http://dx.doi. org/10.1016/0263-2373(95)00005-6

Kim, Y. G., Kim, J. W., Shin, S. 0., \& Baik, D. K. (2006). Managing variability for software product-line. In Proceedings of the 4th International Conference on Software Engineering Research, Management and Applications, Seattle.

Klopffer, W. (2006). The role of SETAC in the development of LCA. International Journal of Life Cycle Assessment, 11(1), 116-122. http://dx.doi.org/10.1065/ lca2006.04.019

Kouskouras, K. G., \& Georgiou, A. C. (2007). A discrete event simulation model in the case of managing a software project. European Journal of Operational Research, 181(1), 374-389. http://dx.doi.org/10.1016/j. ejor.2006.05.031

Krause, F. L., Hayka, H., \& Pasewaldt, B. (2006). Efficient product data sharing in collaboration life cycles. In Proceedings of the 14th International CIRP Design Seminar, Cairo, Egypt.

Kruse, S. A., Flysjo, A., Kasperczyk, N., \& Scholz, A. J. (2009). Socioeconomic indicators as a complement to life cycle assessment-an application to salmon production systems. International Journal of Life Cycle Assessment, 14(1), 8-18. http://dx.doi.org/10.1007/ s11367-008-0040-x

Kurk, F., \& Eagan, P. (2008). The value of adding designfor-the-environment to pollution prevention assistance options. Journal of Cleaner Production, 16(6), 722-726. http://dx.doi.org/10.1016/j.jclepro.2007.02.022

Lee, H. M., Lu, W. F., Song, B., \& Gay, R. (2007). EOL framework for design advisory. In Proceedings of the 4th International Conference on Product Lifecycle Management, Stezzano, Italy.

Li, J. T., \& Kozhikode, R. K. (2009). Developing new innovation models: Shifts in the innovation landscapes in emerging economies and implications for global R\&D manage. Journal of International Management, 15(3), 328-339. http://dx.doi.org/10.1016/j.intman.2008.12.005

Li, M., Qin, X. S., \& Xu, Y. B. (2008). An integrated modeling method supporting product development process optimization. In Proceedings of the 2nd International Conference on Research and Practical lssues of Enterprise Information Systems, Beijing, China.

Lin, J., Qian, Y. J., Cui, W. T., \& Miao, Z. L. (2010). Overlapping and communication policies in product development. European Journal of Operational Research, 201(3), 737750. http://dx.doi.org/10.1016/j.ejor.2009.03.040

Lindahl, M., Skoglund, L., Svensson, J., \& Karlsson, R. (2003). Use and perception of design for environment in small and medium sized enterprises in Sweden. In Proceedings of the 3rd International Symposium on Environmentally Conscious Design and Inverse Manufacturing, Tokyo, Japan.
Linton, J. D., Klassen, R., \& Jayaraman, V. (2007). Sustainable supply chains: An introduction. Journal of Operations Management, 25(6), 1075-1082. http://dx.doi. org/10.1016/j.jom.2007.01.012

Littell, J. H., Corcoran, J., \& Pillai, V. (2008). Systematic Reviews and Meta-Analysis. New York: Oxford University Press, lnc. http://dx.doi.org/10.1093/acprof:0 so/9780195326543.001.0001

Lopes, A. P. V. V., \& Carvalho, M. M. (2012). Evolução da literatura de inovação em relações de cooperação: um estudo bibliométrico num período de vinte anos. Gestão \& Produção, 19(1), 203-217. http://dx.doi.org/10.1590/ S0104-530X2012000100014

Lu, 1. Y., Yang, C. Y., \& Tseng, C. J. (2009). Push-Pull interactive model of service innovation cycle - under the service encounter framework. African Journal of Business Management, 3(9), 433-442.

Lundteigen, M. A., Rausand, M., \& Utne, I. B. (2009). Integrating RAMS engineering and management with the safety life cycle of IEC 61508. Reliability Engineering \& System Safety, 94(12), 1894-1903. http://dx.doi. org/10.1016/j.ress.2009.06.005

Luttropp, C., \& Lagerstedt, J. (2006). Ecodesign and the ten golden rules: generic advice for merging environmental aspects into product development. Journal of Cleaner Production, 14(15-16), 1396-1408. http://dx.doi. org/10.1016/j.jclepro.2005.11.022

Martín, L. Á. G., Cabestre, F. J. R., \& Vega, A. V. R. (1999) El estado actual de la investigación empírica sobre economía de la empresa: análisis de las publicaciones españlas. Papeles de economía española, (78-79), 302317.

Maxwell, D., Sheate, W., \& Van Der Vorst, R. (2006) Functional and systems aspects of the sustainable product and service development approach for industry. Journal of Cleaner Production, 14(17), 1466-1479. http://dx.doi.org/10.1016/j.jclepro.2006.01.028

Mengoni, M., Germani, M., \& Mandorli, F. (2009). A structured agile design approach to support customization in wellness product development. International Journal of Computer Integrated Manufacturing, 22(1), 42-54. http://dx.doi.org/10.1080/09511920802326233

Mulder, K. F. (2007). Innovation for sustainable development: from environmental design to transition management. Sustainability Science, 2(2), 253-263. http://dx.doi. org/10.1007/s11625-007-0036-7

Nakamura, S., \& Kondo, Y. (2006). A waste input-output lifecycle cost analysis of the recycling of end-of-life electrica home appliances. Ecological Economics, 57(3), 494-506. http://dx.doi.org/10.1016/j.ecolecon.2005.05.002

Noel, F., \& Roucoules, L. (2008). The PPO design model with respect to digital enterprise technologies among product life cycle. International Journal of Computer Integrated Manufacturing, 21(2), 139-145. http://dx.doi. org/10.1080/09511920701607782

Norris, G. A. (2006). Social impacts in product life cycles - Towards life cycle attribute assessment. International Journal of Life Cycle Assessment, 11(1), 97104. http://dx.doi.org/10.1065/lca2006.04.017

Ntiamoah, A., \& Afrane, G. (2009). Life cycle assessment of chocolate produced in Ghana. In Proceedings of the International Conference on Environmental Research, Technology and Policy, Ghana, South Africa. 
Ny, H., MacDonald, J. P., Broman, G., Yamamoto, R., \& Robert, K. H. (2006). Sustainability constraints as system boundaries - An approach to making lifecycle management strategic. Journal of Industrial Ecology, 10(1/2), 61-77.

Partidario, P. J., Lambert, J., \& Evans, S. (2007). Building more sustainable solutions in production-consumption systems: the case of food for people with reduced access. Journal of Cleaner Production, 15(6), 513-524. http:// dx.doi.org/10.1016/j.jclepro.2006.05.011

Pero, M., \& Sianesi, A. (2007). Aligning supply chain management and new product development: a general framework. In Proceedings of the 4th International Conference on Product Lifecycle Management, Stezzano, italy.

Pujari, D. (2006). Eco-innovation and new product development: understanding the influences on market performance. Technovation, 26(1), 76-85. http://dx.doi. org/10.1016/j.technovation.2004.07.006

Rausand, M., \& Utne, 1. B. (2009). Product safety - Principles and practices in a life cycle perspective. Safety Science, 47(7), 939-947. http://dx.doi.org/10.1016/j. ssci.2008.10.004

Razavian, M., \& Khosravi, R. (2008). Modeling variability in business process models using UML. 5th International Conference on Information Technology - New Generations, Las Vegas, NV, Apr), 82-87.

Rosenstrom, U., \& Kyllonen, S. (2007). Impacts of a participatory approach to developing national level sustainable development indicators in Finland. Journal of Environmental Management, 84(3), 282-298. http:// dx.doi.org/10.1016/j.jenvman.2006.06.008

Rouibah, K., \& Ould-Ali, S. (2007). Dynamic data sharing and security in a collaborative product definition management system. Robotics and Computer-Integrated Manufacturing, 23(2), 217-233.

Rozenfeld, H., Forcellini, F. A., Amaral, D. C., Toledo, J. C., Silva, S. L., Alliprandini, D. H., Scalice, R. K. (2006). Gestão de Desenvolvimento de Produtos: uma referência para a melhoria do processo. São Paulo: Saraiva.

Salvioni, C. (2007). Feedback and communication: the central role of the user during all the life-cycle of a technical product. Case study from a higher education intranet system. In Proceedings of the International MultiConference on Society, Cybernetics and Informatics, Orlando.

Santos, M. V., \& Bastos, A. V. B. (2007). Redes sociais informais e compartilhamento de significados sobre mudança organizacional. RAE, 47(3), 27-39.

Schatten, A. (2009). Green supply chains: using information integration for sustainable development. In Proceedings of the 3th International Conference on Complex, Intelligent and Software Intensive Systems, Fukuoka, Japan.

Schildt, H. A. (2002). Sitkis: Software for bibliometric data management and analysis. Helsinki: Institute of Strategy and International Business; v. 6. 1.

Schmidt, W. P., \& Butt, F. (2006). Life cycle tools within Ford of Europe's Product Sustainability Index - Case study ford S-MAX \& Ford Galaxy. International Journal of Life Cycle Assessment, 11(5), 315-326. http://dx.doi. org/10.1065/lca2006.08.267
Shao, X. Y., Wu, J., Deng, C., Li, P. G., \& Feng, C. X. J. (2006). A web-enabled collaborative quality management system. Journal of Manufacturing Systems, 25(2), 25-107.

Souza, P. F. D., \& Pereira, H. B. D. (2006). Towards indicators of sustainable product design. In Proceedings of the IEEE International Engineering Management Conference, Salvador, Brazil.

Subramoniam, R., Huisingh, D., \& Chinnam, R. B. (2009). Remanufacturing for the automotive aftermarketstrategic factors: literature review and future research needs. Journal of Cleaner Production, 17(13), 11631174. http://dx.doi.org/10.1016/j.jclepro.2009.03.004

Taifi, N. (2008). Collaborative knowledge networks - Lessons to learn from a large automotive company. Joint Conference of the International-Federation-for-InformationProcessing Working Group. 3rd International Summer School on the Future of Identity in the Information Society, Karlstad, Sweden), 423-429.

Tang, D. B., \& Qian, X. M. (2008). Product lifecycle management for automotive development focusing on supplier integration. Computers in Industry, 59(2/3), 288295. http://dx.doi.org/10.1016/j.compind.2007.07.002

Tao, L., Probert, D., \& Phaal, R. (2010). Towards an integrated framework for managing the process of innovation. $R \& D$ Management, 4O(1), 19-30. http://dx.doi.org/10.1111/ j.1467-9310.2009.00575.x

Tingstrom, J., Swanstrom, L., \& Karlsson, R. (2006). Sustainability management in product development projects - the ABB experience. Journal of Cleaner Production, 14(15-16), 1377-1385. http://dx.doi. org/10.1016/j.jclepro.2005.11.027

Tomovic, C., Anderson, H., Anglin, A., Barreto, L. V., Frillman, S. A., Georgiades, T. J., Homan, S. R., Kochert, J. F., Lech, M. B., Sukup, J. E., Wilde, K. L., \& Wisma, M. (2009). Social issues of product lifecycle management: developing cross cultural virtual teams; supporting today's green manufacturing imperative; educating and preparing tomorrow's workforce; and impacting interorganizational relationships in supply chain management. In Proceedings of the International Conference on Comprehensive Product Realization, Beijing, China.

Vachon, S., \& Klassen, R. D. (2008). Environmental management and manufacturing performance: the role of collaboration in the supply chain. International Journal of Production Economics, 111(2), 299-315. http://dx.doi.org/10.1016/j.ijpe.2006.11.030

Van Berkel, R. (2007). Eco-efficiency in primary metals production: context, perspectives and methods. Resources Conservation and Recycling, 51(3), 511-540. http://dx.doi.org/10.1016/j.resconrec.2007.03.007

Van Schaik, A., Reuter, M. A., \& Van Stokkom, H. (2010). Management of the web of water and web of materials. Minerals Engineering, 23(3), 157-174. http://dx.doi. org/10.1016/j.mineng.2009.08.009

Veshagh, A., \& Obagun, A. (2007). Survey of sustainable life cycle design and management. 14th CIRP Conference on Life Cycle Engineering, Tokyo, Japan, Jun), 237-242.

Vezzoli, C., \& Sciama, D. (2006). Life cycle design: from general methods to product type specific guidelines and checklists: a method adopted to develop a set of guidelines/checklist handbook for the eco-efficient design of NECTA vending machines. Journal of Cleaner Production, 14(15-16), 1319-1325. http://dx.doi. org/10.1016/j.jclepro.2005.11.011 
Waage, S. A. (2007). Re-considering product design: a practical "road-map" for integration of sustainability issues. Journal of Cleaner Production, 15(7), 638-649. http://dx.doi.org/10.1016/j.jclepro.2005.11.026

Wang, S. Y., Chang, S. L., \& Wang, R. C. (2009). Assessment of supplier performance based on product-development strategy by applying multi-granularity linguistic term sets. Omega-International Journal of Management Science, 37(1), 215-226. http://dx.doi.org/10.1016/j. omega.2006.10.003

Yang, Q. Z., Miao, C. Y., Zhang, Y., \& Gay, R. (2006). LCA and LCC data semantics sharing across product lifecycle processes. In Proceedings of the 13th ISPE International Conference on Concurrent Engineering, Antibes, France.

Zhang, H. J., \& Huang, H. W. (2006). Reverse logistics system of waste electronic and electric equipment.
In Proceedings of the International Conference on Management of Logistics, Sydney, Australia.

\section{Agradecimentos}

Os autores agradecem à CAPES pelo apoio à presente pesquisa, por meio do Procad, e também aos avaliadores da Revista Production pela contribuição significativa para a melhoria do presente trabalho. Um dos autores também tem vínculo com o Departamento de Engenharia de Produção e Sistemas da Universidade Federal de Santa Catarina - UFSC e, por essa razão, essa instituição também merece agradecimentos.

\title{
Product life cycle management and product development: biblio- metric analysis and literature classification
}

\begin{abstract}
The aim of this paper is to present a bibliometric analysis and classification of the literature on the concepts Product Life Cycle Management (PLM), New Product Development (NPD), Environmental Sustainability (ES) and their interfaces. The ISI Web of Knowledge database was accessed, as was the CAPES Brazilian portal, which is linked to various databases. The analysis considers publications between 2006 and 2010. The results show that papers are published in a dispersed way in different journals. The majority of the papers adopt case studies as a methodological research approach, but there are also many theoretical-based papers. Concerning the nature of the data, qualitative approaches are more commonly used than are quantitative ones, and most papers consider descriptive studies. One of the reasons that qualitative approaches are used more frequently may be that these concepts are not well consolidated in the literature. The examined publications incorporated the concept of ES and PLM in NPD and are more extensively applied in industries, supply chain and software development. In general, the publications emphasize PDP improvement, performance assessment and the integration of information from different areas. Finally, the article concludes that the literature converges to an integration of the concepts of environmental sustainability and PLM within the current practices of NPD.
\end{abstract}

\section{Keywords}

Environmental sustainability (ES). Product life cycle management (PLM). New product development (NPD). 
Apêndice 1. Codificação da classificação dos trabalhos sobre Product Life Cycle Management, Product Development Process e Sustentabilidade.

\begin{tabular}{cc}
\hline \multicolumn{2}{c}{ T1 - Tipo de estudo } \\
\hline MO & Modelagem \\
TC & Teórico-conceitual \\
RL & Revisão da literatura \\
SI & Simulação \\
SU & Survey \\
EC & Estudo de caso \\
PA & Pesquisa-ação \\
EX & Experimental \\
\hline
\end{tabular}

\begin{tabular}{ccc}
\hline & \multicolumn{2}{c}{ T7 - Documentação } \\
\hline & QU & Questionário \\
& EN & Entrevistas \\
& AD & Análise documental \\
& Dados públicos \\
& DP & Revisão bibliográfica \\
& B1 & Observação \\
\hline & OB & \\
\hline & T8 - Aplicação \\
\hline A1 & Ambientes complexos \\
A2 & Cadeia de demanda \\
A3 & Cadeia de suprimentos \\
A4 & Codesenvolvimento \\
A5 & Processo de desenvolvimento de produto \\
A6 & Programas de educação \\
A7 & Software \\
A8 & Tecnologia \\
\hline
\end{tabular}

\begin{tabular}{ccc}
\hline & T2 - Apoio financeiro & \\
\hline $\mathrm{S}$ & Sim \\
$\mathrm{N}$ & Não \\
\hline
\end{tabular}

\begin{tabular}{cc}
\hline \multicolumn{2}{c}{ T3 - Período de análise } \\
\hline LO & Longitudinal \\
RE & Retrospectivo \\
AT & Atual \\
\hline
\end{tabular}

\begin{tabular}{cccc}
\hline \multicolumn{4}{c}{ T4 - Abordagem } \\
\hline \multirow{2}{*}{ QT } & Quantitativo & $\mathrm{d}$ & Descritivo \\
& & $\mathrm{p}$ & Preditivo \\
QL & Qualitativo & $\mathrm{d}$ & Descritivo \\
& & $\mathrm{p}$ & Preditivo \\
\hline
\end{tabular}

\begin{tabular}{cc}
\hline & T9 - Benefícios \\
\hline B1 & Alinhamento \\
B2 & Desempenho \\
B3 & Integrar informações \\
B4 & Melhorar controle e planejamento \\
B5 & Metodologia \\
B6 & Sustentabilidade \\
B7 & Tomada de decisão \\
\hline & T10 - Conceitos \\
\hline C1 & Sustentabilidade \\
C2 & Product Development Process (PDP) \\
C3 & Product Life Cycle Management (PLM) \\
\hline
\end{tabular}

\begin{tabular}{cc}
\hline \multicolumn{3}{c}{ T5 - Abrangência geográfica } \\
\hline RE & Regional \\
NA & Nacional \\
IN & Internacional \\
\hline \multicolumn{3}{c}{ T6 - Unidade de análise } \\
\hline PE & Pessoas \\
GR & Grupos \\
UO & Unidade organizacional \\
EM & Empresas \\
\hline
\end{tabular}


Apêndice 2. Apresentação dos trabalhos codificados em ordem alfabética.

\begin{tabular}{|c|c|c|c|c|c|c|c|c|c|c|}
\hline Artigos & $\mathrm{T} 1$ & $\mathrm{~T} 2$ & T3 & $\mathrm{T} 4$ & T5 & T6 & T7 & T8 & T9 & T10 \\
\hline Aurich et al. (2007) & EC & $\mathrm{S}$ & RE & $\mathrm{QL} / \mathrm{d}$ & NA & EM & $\mathrm{AD}$ & $\mathrm{A} 1$ & B6 & $\mathrm{C} 1 / \mathrm{C} 3$ \\
\hline Baccile et al. (2009) & $\mathrm{RL}$ & $\mathrm{N}$ & RE & $\mathrm{QL} / \mathrm{d}$ & NA & GR & $\mathrm{Bl}$ & A5 & B2 & $\mathrm{C} 1 / \mathrm{C} 2$ \\
\hline Barry et al. (2006) & MO & $S$ & lo & $\mathrm{QT} / \mathrm{d}$ & NA & EM & $\mathrm{OB} / \mathrm{AD} / \mathrm{Bl}$ & A7 & B7 & $\mathrm{C} 1 / \mathrm{C} 3$ \\
\hline Belkadi et al. (2008) & EC & $\mathrm{N}$ & AT & $\mathrm{QL} / \mathrm{d}$ & NA & EM & $\mathrm{AD} / \mathrm{B} 1$ & A3 & B2 & $\mathrm{C} 3$ \\
\hline Ben Mahmoud-Jouini \& Lenfle (2010) & EC & $\mathrm{N}$ & RE & $\mathrm{QL} / \mathrm{d}$ & NA & EM & EN/AD & $\mathrm{A} 1$ & B2 & C3 \\
\hline Bergea et al. (2006) & $\mathrm{TC}$ & $\mathrm{N}$ & RE & $\mathrm{QL} / \mathrm{d}$ & NA & uo & OB & A5 & B5 & $\mathrm{C} 1 / \mathrm{C} 2$ \\
\hline Bertolini et al. (2007) & SU/EC & $\mathrm{S}$ & RE & $\mathrm{QT} / \mathrm{d}$ & NA & EM & QU/EN & $\mathrm{A} 1$ & B7 & $\mathrm{C} 2$ \\
\hline Bidokhti (2008) & $\mathrm{TC}$ & $\mathrm{N}$ & RE & $\mathrm{QL} / \mathrm{d}$ & NA & EM & $A D$ & A5 & B2 & $\mathrm{C} 3$ \\
\hline Bordoloi \& Guerrero (2008) & TC & $\mathrm{N}$ & AT & $\mathrm{QL} / \mathrm{d}$ & NA & EM & $\mathrm{Bl}$ & A7 & B3 & $\mathrm{C} 3$ \\
\hline Broy (2009) & MO & $\mathrm{N}$ & AT & $\mathrm{QL} / \mathrm{d}$ & NA & EM & $\mathrm{AD} / \mathrm{Bl}$ & A5 & B3 & $\mathrm{C} 3$ \\
\hline Buganza \& Verganti (2006) & $\mathrm{EC} / \mathrm{SU}$ & $\mathrm{N}$ & RE & QT-QL/d & NA & EM & $\mathrm{QU} / \mathrm{EN} / \mathrm{AD} / \mathrm{OB}$ & $\mathrm{A} 1$ & B2 & $\mathrm{C} 2$ \\
\hline Cebon et al. (2008) & $\mathrm{TC}$ & $\mathrm{S}$ & AT & $\mathrm{QL} / \mathrm{d}$ & $1 \mathrm{~N}$ & EM & $\mathrm{Bl}$ & $\mathrm{A} 1$ & B2 & $\mathrm{C} 2 / \mathrm{C} 3$ \\
\hline Chang et al. (2007) & $\mathrm{EC}$ & $\mathrm{S}$ & RE & $\mathrm{QL} / \mathrm{d}$ & NA & EM & $\mathrm{Bl}$ & $\mathrm{A} 1$ & B2 & $\mathrm{C} 2 / \mathrm{C} 3$ \\
\hline Ciolkowski \& Soto (2008) & $\mathrm{EC} / \mathrm{MO}$ & $\mathrm{S}$ & lo & $\mathrm{QL} / \mathrm{d}$ & NA & EM & EN & $\mathrm{A} 1$ & B5 & $\mathrm{C} 1 / \mathrm{C} 3$ \\
\hline Cooper et al. (2008) & MO & $\mathrm{N}$ & AT & $\mathrm{QL} / \mathrm{d}$ & NA & EM & $\mathrm{Bl}$ & A7 & B7 & $\mathrm{C} 2 / \mathrm{C} 3$ \\
\hline Cooper (2007) & $\mathrm{TC}$ & $\mathrm{N}$ & RE & $\mathrm{QL} / \mathrm{d}$ & $\mathrm{RE}$ & vo & DP & A6 & B5 & $\mathrm{C} 1$ \\
\hline Cordella et al. (2008) & $\mathrm{EC}$ & $\mathrm{S}$ & RE & $\mathrm{QT} / \mathrm{d}$ & NA & EM & $\mathrm{Bl} / \mathrm{AD}$ & $\mathrm{A} 1$ & B2 & $\mathrm{C} 1$ \\
\hline Da Silva et al. (2008) & EC & $\mathrm{S}$ & AT & $\mathrm{QT} / \mathrm{d}$ & NA & $\mathrm{GR}$ & $\mathrm{Bl} / \mathrm{AD}$ & A7 & B6 & $\mathrm{C} 2 / \mathrm{C}_{3}$ \\
\hline De Benedetto \& Klemes (2009) & EC & $\mathrm{S}$ & RE & QT/d & NA & EM & $\mathrm{AD}$ & $\mathrm{A} 1$ & B7 & $\mathrm{C} 1 / \mathrm{C}_{3}$ \\
\hline Dehghanian \& Mansour (2008) & $\mathrm{MO} / \mathrm{EC}$ & $\mathrm{N}$ & AT & $\mathrm{QT} / \mathrm{d}$ & NA & EM & $\mathrm{AD} / \mathrm{Bl}$ & A3 & B7 & $\mathrm{C} 1$ \\
\hline Donnelly et al. (2006) & $\mathrm{EC}$ & $\mathrm{N}$ & RE & $\mathrm{QL} / \mathrm{d}$ & NA & EM & $\mathrm{AD}$ & A7 & B3 & $\mathrm{C} 1 / \mathrm{C}_{2} / \mathrm{C}_{3}$ \\
\hline Ebert \& De Man (2008) & EC & $\mathrm{N}$ & RE & $\mathrm{QL} / \mathrm{d}$ & NA & EM & $\mathrm{AD} / \mathrm{Bl}$ & A5 & B5 & C3 \\
\hline Elghali et al. (2008) & TC & $\mathrm{N}$ & RE & $\mathrm{QL} / \mathrm{d}$ & NA & EM & $\mathrm{Bl}$ & $\mathrm{A} 1$ & B7 & $\mathrm{C} 1 / \mathrm{C} 2$ \\
\hline Eun et al. (2009) & EC & $\mathrm{N}$ & lo & QT/d & NA & vo & $\mathrm{Bl} / \mathrm{AD}$ & A7 & B3 & $\mathrm{C} 1$ \\
\hline Fargnoli \& Kimura (2006) & $\mathrm{S} 1$ & $\mathrm{~S}$ & Lo & $\mathrm{QT} / \mathrm{d}$ & NA & EM & $\mathrm{AD} / \mathrm{Bl} / \mathrm{DP}$ & A5 & B5 & $\mathrm{C} 1 / \mathrm{C} 2$ \\
\hline Fixson (2007) & $\mathrm{RL}$ & $\mathrm{N}$ & RE & $\mathrm{QL} / \mathrm{d}$ & NA & vo & $\mathrm{Bl}$ & $\mathrm{A} 1$ & B5 & $\mathrm{C} 3$ \\
\hline Genaidy \& Karwowski (2008) & $\mathrm{TC}$ & $\mathrm{N}$ & AT & $\mathrm{QL} / \mathrm{d}$ & NA & EM & $\mathrm{Bl}$ & A8 & B6 & $\mathrm{C} 2 / \mathrm{C}_{3}$ \\
\hline Giudice et al. (2009) & $\mathrm{MO} / \mathrm{EC}$ & $\mathrm{N}$ & AT & $\mathrm{QL} / \mathrm{d}$ & NA & uo & $\mathrm{AD}$ & A7 & B2 & $\mathrm{C} 1 / \mathrm{C} 2$ \\
\hline Han \& Do (2006) & MO & $\mathrm{N}$ & AT & $\mathrm{QL} / \mathrm{d}$ & NA & EM & $\mathrm{OB} / \mathrm{AD} / \mathrm{B} 1$ & A5 & B7 & $\mathrm{C} 2 / \mathrm{C}_{3}$ \\
\hline Hauschild et al. (2008) & $\mathrm{TC}$ & $\mathrm{N}$ & lo & $\mathrm{QL} / \mathrm{d}$ & NA & EM & $\mathrm{Bl} / \mathrm{AD}$ & A3 & B5 & $\mathrm{C} 1$ \\
\hline Hobby et al. (2009) & $\mathrm{TC}$ & $\mathrm{N}$ & AT & $\mathrm{QL} / \mathrm{d}$ & NA & EM & $\mathrm{Bl}$ & A8 & B2 & $\mathrm{C} 1$ \\
\hline Hochschorne \& Finnveden (2006) & EC & $\mathrm{S}$ & $\mathrm{RE}$ & $\mathrm{QT} / \mathrm{d}$ & NA & $\mathrm{GR}$ & EN & A8 & B6 & $\mathrm{C} 1$ \\
\hline Huang (2009) & MO & $\mathrm{N}$ & AT & $\mathrm{QL} / \mathrm{d}$ & $1 \mathrm{~N}$ & EM & $\mathrm{DP} / \mathrm{B} 1$ & A7 & B6 & $\mathrm{C} 1 / \mathrm{C} 2$ \\
\hline Jawahir et al. (2007) & $\mathrm{EC}$ & $\mathrm{N}$ & RE & $\mathrm{QL} / \mathrm{d}$ & NA & vo & $\mathrm{OB} / \mathrm{AD} / \mathrm{Bl}$ & A6 & B5 & $\mathrm{C} 1 / \mathrm{C} 2$ \\
\hline Jiang et al. (2008) & EC & $\mathrm{S}$ & AT & $\mathrm{QL} / \mathrm{d}$ & NA & EM & $\mathrm{AD} / \mathrm{Bl}$ & A7 & B3 & $\mathrm{C} 1$ \\
\hline Joore (2008) & EC & $\mathrm{N}$ & RE & $\mathrm{QL} / \mathrm{d}$ & NA & EM & $\mathrm{EN} / \mathrm{AD} / \mathrm{OB}$ & $\mathrm{A} 1$ & B5 & $\mathrm{C} 1 / \mathrm{C} 2$ \\
\hline Juttner et al. (2006) & EC & $\mathrm{S}$ & RE & $\mathrm{QL} / \mathrm{d}$ & NA & EM & $\mathrm{EN} / \mathrm{Bl} / \mathrm{AD}$ & $\mathrm{A} 2$ & B1 & $\mathrm{C} 3$ \\
\hline Kennedy et al. (2007) & EC & $\mathrm{N}$ & RE & $\mathrm{QL} / \mathrm{d}$ & NA & EM & $\mathrm{QU} / \mathrm{EN} / \mathrm{AD}$ & $\mathrm{A} 1$ & B5 & $\mathrm{C} 2 / \mathrm{C} 3$ \\
\hline Khota \& Pretorius (2008) & TC & $\mathrm{N}$ & AT & $\mathrm{QL} / \mathrm{d}$ & NA & EM & $\mathrm{Bl}$ & A5 & B2 & $\mathrm{C} 2$ \\
\hline Kim et al. (2006) & $\mathrm{EC} / \mathrm{MO}$ & $\mathrm{S}$ & Lo & $\mathrm{QL} / \mathrm{d}$ & NA & EM & $\mathrm{AD} / \mathrm{Bl}$ & $\mathrm{A} 1$ & B5 & $\mathrm{C} 2 / \mathrm{C} 3$ \\
\hline Klopffer (2006) & TC & $\mathrm{N}$ & RE & $\mathrm{QL} / \mathrm{d}$ & NA & $\mathrm{GR}$ & $\mathrm{Bl}$ & $\mathrm{A} 1$ & B5 & $\mathrm{C} 1$ \\
\hline Kouskouras \& Georgiou (2007) & $\mathrm{MO} / \mathrm{Sl}$ & $\mathrm{N}$ & AT & $\mathrm{QT} / \mathrm{d}$ & NA & EM & $\mathrm{AD} / \mathrm{Bl}$ & A7 & B4 & $\mathrm{C} 3$ \\
\hline Krause et al. (2006) & MO & $\mathrm{S}$ & AT & $\mathrm{QL} / \mathrm{d}$ & NA & EM & $\mathrm{AD} / \mathrm{Bl}$ & A5 & B5 & $\mathrm{C} 3$ \\
\hline Kruse et al. (2009) & $\mathrm{TC}$ & $\mathrm{N}$ & RE & $\mathrm{QL} / \mathrm{d}$ & NA & EM & $\mathrm{AD} / \mathrm{B} 1$ & $\mathrm{~A} 1$ & B5 & $\mathrm{C} 1 / \mathrm{C} 3$ \\
\hline Kurk \& Eagan (2008) & $\mathrm{TC}$ & $\mathrm{N}$ & AT & $\mathrm{QL} / \mathrm{d}$ & NA & vo & $\mathrm{Bl}$ & $\mathrm{A} 1$ & B5 & $\mathrm{C} 1 / \mathrm{C} 2$ \\
\hline Lee et al. (2007) & MO & $\mathrm{N}$ & AT & $\mathrm{QL} / \mathrm{d}$ & NA & EM & $\mathrm{Bl}$ & A7 & B5 & $\mathrm{C} 3$ \\
\hline Li \& Kozhikode (2009) & $\mathrm{TC}$ & $\mathrm{S}$ & $\mathrm{RE}$ & $\mathrm{QL} / \mathrm{d}$ & $\mathrm{IN}$ & EM & $\mathrm{Bl} / \mathrm{DP}$ & $\mathrm{A} 1$ & B5 & $\mathrm{C} 2 / \mathrm{C} 3$ \\
\hline Li et al. (2008) & EC & $\mathrm{S}$ & RE & $\mathrm{QL} / \mathrm{d}$ & NA & EM & $\mathrm{AD} / \mathrm{Bl}$ & A7 & B3 & $\mathrm{C} 2 / \mathrm{C}_{3}$ \\
\hline Lin et al. (2010) & $\mathrm{MO} / \mathrm{EC}$ & $\mathrm{N}$ & AT & $\mathrm{QT} / \mathrm{d}$ & NA & EM & $\mathrm{EN} / \mathrm{AD}$ & $\mathrm{A} 1$ & B2 & $\mathrm{C} 2$ \\
\hline Linton et al. (2007) & $\mathrm{RL}$ & $\mathrm{N}$ & $\mathrm{RE}$ & $\mathrm{QT} / \mathrm{d}$ & $\mathrm{IN}$ & $\mathrm{GR}$ & $\mathrm{Bl}$ & A3 & B5 & $\mathrm{C} 1 / \mathrm{C}_{3}$ \\
\hline Lu et al. (2009) & EC & $\mathrm{S}$ & AT & QT-QL/d & NA & EM & QU/EN & $\mathrm{A} 1$ & B2 & $\mathrm{C} 2$ \\
\hline Lundteigen et al. (2009) & $\mathrm{MO} / \mathrm{TC}$ & $\mathrm{N}$ & AT & $\mathrm{QL} / \mathrm{d}$ & NA & vo & $\mathrm{AD} / \mathrm{B} 1$ & $\mathrm{~A} 1$ & B5 & $\mathrm{C} 2 / \mathrm{C} 3$ \\
\hline Luttropp \& Lagerstedt (2006) & EC & $\mathrm{N}$ & RE & $\mathrm{QL} / \mathrm{d}$ & NA & EM & $\mathrm{AD} / \mathrm{Bl}$ & A5 & B3 & $\mathrm{C} 1 / \mathrm{C} 2$ \\
\hline Maxwell et al. (2006) & EC & $\mathrm{N}$ & lo & $\mathrm{QT} / \mathrm{d}$ & NA & EM & $\mathrm{QU} / \mathrm{AD}$ & A5 & B2 & $\mathrm{C} 1 / \mathrm{C} 2$ \\
\hline Mengoni et al. (2009) & EC & $\mathrm{N}$ & AT & $\mathrm{QL} / \mathrm{d}$ & NA & EM & $\mathrm{AD}$ & A5 & B3 & $\mathrm{C} 1 / \mathrm{C} 2$ \\
\hline
\end{tabular}

T1: Tipo de estudo; T2: Apoio financeiro; T3: Período de análise; T4: Abordagens; T5: Abrangência geográfica; T6: Unidade de análise; T7: Documentação; T8: Aplicação; T9: Benefícios; T10: Conceitos. 
Apêndice 2. Continuação...

\begin{tabular}{|c|c|c|c|c|c|c|c|c|c|c|}
\hline Artigos & $\mathrm{T} 1$ & $\mathrm{~T} 2$ & T3 & T4 & T5 & T6 & T7 & T8 & T9 & $\mathrm{T} 10$ \\
\hline Mulder (2007) & $\mathrm{TC}$ & $\mathrm{N}$ & $\mathrm{RE}$ & $\mathrm{QL} / \mathrm{d}$ & NA & EM & $\mathrm{B} 1$ & A8 & B2 & $\mathrm{C} 1$ \\
\hline Nakamura \& Kondo (2006) & EC & $\mathrm{S}$ & RE & $\mathrm{QT} / \mathrm{d}$ & NA & EM & $\mathrm{AD} / \mathrm{Bl} / \mathrm{DP}$ & $\mathrm{A} 1$ & B7 & C3 \\
\hline Noel \& Roucoules (2008) & TC & $\mathrm{N}$ & AT & $\mathrm{QL} / \mathrm{d}$ & NA & EM & $\mathrm{Bl}$ & $\mathrm{A} 1$ & B5 & $\mathrm{C} 2 / \mathrm{C} 3$ \\
\hline Norris (2006) & EC & $\mathrm{S}$ & RE & $\mathrm{QL} / \mathrm{d}$ & $1 \mathrm{~N}$ & EM & $\mathrm{AD} / \mathrm{B} 1$ & $\mathrm{~A} 1$ & B5 & $\mathrm{C} 1 / \mathrm{C} 2 / \mathrm{C} 3$ \\
\hline Ntiamoah \& Afrane (2009) & EC & $\mathrm{N}$ & RE & $\mathrm{QT} / \mathrm{d}$ & NA & $\mathrm{GR}$ & EN/DP & $\mathrm{A} 1$ & B6 & $\mathrm{C} 1$ \\
\hline Ny et al. (2006) & $\mathrm{TC}$ & $\mathrm{S}$ & RE & $\mathrm{QL} / \mathrm{d}$ & NA & EM & $\mathrm{Bl}$ & A5 & B5 & $\mathrm{C} 1 / \mathrm{C}_{3}$ \\
\hline Partidario et al. (2007) & EC & $\mathrm{N}$ & RE & $\mathrm{QL} / \mathrm{d}$ & NA & EM & $\mathrm{EN} / \mathrm{AD} / \mathrm{OB}$ & A4 & B5 & $\mathrm{C} 1 / \mathrm{C} 2$ \\
\hline Pero \& Sianesi (2007) & EC & $\mathrm{N}$ & RE & $\mathrm{QL} / \mathrm{d}$ & NA & EM & $\mathrm{EN} / \mathrm{AD} / \mathrm{OB}$ & A3 & B3 & $\mathrm{C} 2$ \\
\hline Pujari (2006) & SU & $\mathrm{N}$ & AT & $\mathrm{QT} / \mathrm{d}$ & NA & EM & QU/EN & A5 & B2 & $\mathrm{C} 1 / \mathrm{C} 2$ \\
\hline Rausand \& Utne (2009) & EC & $\mathrm{N}$ & AT & $\mathrm{QL} / \mathrm{d}$ & NA & $\mathrm{GR}$ & $\mathrm{AD} / \mathrm{Bl}$ & $\mathrm{A} 1$ & B2 & $\mathrm{C} 3$ \\
\hline Razavian \& Khosravi (2008) & $\mathrm{MO} / \mathrm{EC}$ & $\mathrm{N}$ & AT & $\mathrm{QL} / \mathrm{d}$ & NA & EM & $\mathrm{AD} / \mathrm{Bl}$ & A7 & B3 & $\mathrm{C} 2 / \mathrm{C} 3$ \\
\hline Rosenstrom \& Kyllonen (2007) & EC & $\mathrm{N}$ & RE & $\mathrm{QL} / \mathrm{d}$ & NA & uo & $\mathrm{DP} / \mathrm{Bl} / \mathrm{OB}$ & A5 & B2 & $\mathrm{C} 1$ \\
\hline Rouibah \& Ould-Ali (2007) & $\mathrm{EC}$ & $\mathrm{S}$ & RE & $\mathrm{QT} / \mathrm{d}$ & NA & EM & EN/QU & A4 & B3 & $\mathrm{C} 3$ \\
\hline Salvioni (2007) & EC & $\mathrm{N}$ & RE & $\mathrm{QL} / \mathrm{d}$ & NA & EM & $\mathrm{QU} / \mathrm{AD} / \mathrm{Bl}$ & A8 & B2 & $\mathrm{C} 3$ \\
\hline Schatten (2009) & $\mathrm{TC}$ & $\mathrm{N}$ & RE & $\mathrm{QL} / \mathrm{d}$ & $\mathrm{IN}$ & EM & $\mathrm{Bl} / \mathrm{DP}$ & A3 & B3 & $\mathrm{C} 1 / \mathrm{C} 2$ \\
\hline Schmidt \& Butt (2006) & EC & $\mathrm{N}$ & RE & $\mathrm{QL} / \mathrm{d}$ & NA & EM & $\mathrm{AD} / \mathrm{Bl}$ & $\mathrm{A} 1$ & B2 & $\mathrm{C} 1$ \\
\hline Shao et al. (2006) & $\mathrm{EC} / \mathrm{MO}$ & $\mathrm{S}$ & RE & $\mathrm{QL} / \mathrm{d}$ & NA & EM & $\mathrm{AD} / \mathrm{Bl}$ & A7 & B3 & $\mathrm{C} 3$ \\
\hline Souza \& Pereira (2006) & $\mathrm{TC}$ & $\mathrm{S}$ & RE & $\mathrm{QL} / \mathrm{d}$ & NA & EM & $\mathrm{Bl}$ & A5 & B7 & $\mathrm{C} 1$ \\
\hline Subramoniam et al. (2009) & EC & $\mathrm{N}$ & AT & $\mathrm{QL} / \mathrm{d}$ & NA & EM & EN/AD & $\mathrm{A} 1$ & B5 & $\mathrm{C} 1 / \mathrm{C} 3$ \\
\hline Taifi (2008) & EC & $\mathrm{N}$ & AT & $\mathrm{QL} / \mathrm{d}$ & NA & EM & $\mathrm{EN} / \mathrm{AD} / \mathrm{QU}$ & A4 & B5 & $\mathrm{C} 1 / \mathrm{C} 2$ \\
\hline Tang \& Qian (2008) & EC & $\mathrm{S}$ & RE & $\mathrm{QL} / \mathrm{d}$ & $1 \mathrm{~N}$ & EM & $\mathrm{AD} / \mathrm{Bl}$ & A3 & B2 & $\mathrm{C} 2 / \mathrm{C}_{3}$ \\
\hline Tao et al. (2010) & $\mathrm{EC}$ & $\mathrm{N}$ & AT & $\mathrm{QL} / \mathrm{d}$ & NA & EM & EN & $\mathrm{A} 1$ & B5 & $\mathrm{C} 2$ \\
\hline Tingstrom et al. (2006) & EC & $\mathrm{S}$ & AT & $\mathrm{QL} / \mathrm{d}$ & NA & EM & EN/AD & A5 & B5 & $\mathrm{C} 1 / \mathrm{C} 2$ \\
\hline Tomovic et al. (2009) & $\mathrm{TC}$ & $\mathrm{N}$ & AT & $\mathrm{QL} / \mathrm{d}$ & NA & EM & $\mathrm{Bl}$ & A5 & $\mathrm{B} 2$ & C3 \\
\hline Vachon \& Klassen (2008) & SU & $\mathrm{N}$ & RE & $\mathrm{QT} / \mathrm{d}$ & $\mathrm{IN}$ & EM & QU/EN & A3 & B2 & $\mathrm{C} 1$ \\
\hline Van Berkel (2007) & $\mathrm{TC}$ & $\mathrm{N}$ & RE & $\mathrm{QL} / \mathrm{d}$ & NA & EM & $\mathrm{DP} / \mathrm{B} 1$ & A5 & B2 & $\mathrm{C} 1$ \\
\hline Veshagh \& Obagun (2007) & $\mathrm{EC} / \mathrm{SU}$ & $\mathrm{N}$ & RE & $\mathrm{QL} / \mathrm{d}$ & NA & EM & EN/QU/AD & $\mathrm{A} 1$ & B5 & $\mathrm{C} 1 / \mathrm{C} 2$ \\
\hline Vezzoli \& Sciama (2006) & EC & $\mathrm{N}$ & AT & $\mathrm{QL} / \mathrm{d}$ & NA & EM & $\mathrm{EN} / \mathrm{AD} / \mathrm{Bl}$ & $\mathrm{A} 1$ & B6 & $\mathrm{C} 1$ \\
\hline Waage (2007) & $\mathrm{TC}$ & $\mathrm{N}$ & RE & $\mathrm{QL} / \mathrm{d}$ & NA & EM & $\mathrm{DP} / \mathrm{B} 1$ & $\mathrm{~A} 1$ & B3 & $\mathrm{C} 1 / \mathrm{C} 2$ \\
\hline Wang et al. (2009) & EC & $\mathrm{N}$ & AT & $\mathrm{QT} / \mathrm{d}$ & NA & EM & $\mathrm{AD} / \mathrm{Bl}$ & A3 & B2 & $\mathrm{C} 2$ \\
\hline Yang et al. (2006) & MO & $\mathrm{N}$ & AT & $\mathrm{QL} / \mathrm{d}$ & NA & EM & $\mathrm{AD} / \mathrm{Bl}$ & A7 & B3 & $\mathrm{C} 1 / \mathrm{C} 3$ \\
\hline Zhang \& Huang (2006) & EC & $\mathrm{N}$ & $\mathrm{RE}$ & $\mathrm{QL} / \mathrm{d}$ & NA & EM & $\mathrm{AD} / \mathrm{Bl}$ & A3 & B2 & $\mathrm{C} 1 / \mathrm{C}_{2}$ \\
\hline
\end{tabular}

T1: Tipo de estudo; T2: Apoio financeiro; T3: Período de análise; T4: Abordagens; T5: Abrangência geográfica; T6: Unidade de análise; T7: Documentação; T8: Aplicação; T9: Benefícios; T10: Conceitos. 